\title{
Significant Diagnostic and Prognostic Value of FLAD1 and Related MicroRNAs in Breast Cancer after a Pan-Cancer Analysis
}

\author{
Mei Mei $\mathbb{D}^{1,2}$ Wenting Song, ${ }^{1}$ Yingjun Wang, ${ }^{1}$ and Mingzhi Zhang $\mathbb{D}^{1}$ \\ ${ }^{1}$ Department of Oncology, The First Affiliated Hospital of Zhengzhou University, Zhengzhou, China \\ ${ }^{2}$ The Academy of Medical Sciences, Zhengzhou University, Zhengzhou, China
}

Correspondence should be addressed to Mingzhi Zhang; mingzhi_zhang1@163.com

Received 9 December 2019; Revised 30 May 2021; Accepted 24 June 2021; Published 22 July 2021

Academic Editor: Rudy Foddis Copyright (c) 2021 Mei Mei et al. This is an open access article distributed under the Creative Commons Attribution License, which
permits unrestricted use, distribution, and reproduction in any medium, provided the original work is properly cited.

\begin{abstract}
The identification of biomarkers plays an important role in the diagnosis and prognosis of cancers. In this study, we explored the diagnostic and prognostic value of the FLAD1 expression across pan-cancer analysis from online databases (Oncomine, cBioPortal, Breast Cancer Gene-Expression Miner, UALCAN, GEO, BCIP, TNMplot, ENCORI, Kaplan-Meier Plotter, and LinkedOmics). We found that FLAD1 was overexpressed in a number of different kinds of cancers, especially in breast cancer, and higher FLAD1 expression level was associated with the HER+, p53 mutant, node-involved, NPI stage 3, basal-like, and triple-negative groups compared with the other subgroups of breast cancer. The FLAD1 expression levels were higher in patients that were 21-40 years old than those in patients of other ages and were higher in the African-American group than in the Caucasian group. We also analyzed the FLAD1-related microRNAs and their prognostic values in breast cancer. This study highlights the significance of FLAD1 in cancers and provides evidence for its potential as a biomarker for the diagnosis and prognosis of cancers.
\end{abstract}

\section{Introduction}

Breast cancer is the most common malignancy in women and the most common cause of cancer-related deaths in lessdeveloped countries [1]. Approximately 2.1 million newly diagnosed female breast cancer cases were reported worldwide in 2018 [2]. Breast cancer has various subtypes that differ in histopathology, biology, and response to systemic treatment [3]. Despite the rapid development of new technologies and treatments, the identification of biomarkers for diagnosis and prognosis is still in the early research phase [4].

Flavin adenine dinucleotide synthetase 1 (FLAD1), also known as FAD1, is located on chromosome 1 [5] at 1q21.3 [6] (https://www.genecards.org/). It encodes flavin adenine dinucleotide synthase (FADS), which contains an $\mathrm{N}$ terminal molybdopterin-binding (MPTb) domain and a Cterminal domain sufficient to catalyze FAD synthesis [7]. The FLAD1 expression was previously reported to be upregulated in hepatocellular carcinoma and is considered to be related to hepatitis $B$ virus infection [8]. Another study reported that FLAD1, as well as three other genomic markers,
$D B N 1, C A C N B 3$, and CCND2, could serve as a novel prognostic model of stage I-III non-small-cell lung cancer [9]. The FLAD1 expression has also been shown to be upregulated in gastric cancer [10] and breast cancer [11].

With the development of high-throughput technology, the relationships between oncogene expressions and clinical factors have become obvious. However, the diagnostic and prognostic significance of FLAD1 is unclear. Therefore, in this study, we searched for relevant data from online databases to determine the diagnostic and prognostic value of the abnormal expression of FLAD1 and related miRNAs in pan-cancer analysis, especially in breast cancer. These results have implications for the development of new molecular biomarkers in breast cancer and provide evidence for the clinical value of FLAD1.

\section{Materials and Methods}

2.1. Oncomine Database. Oncomine is an online database [12] (http://www.oncomine.org) with sequencing and bioinformatic data for 715 datasets and 86,733 samples. We 
analyzed FLAD1 in several kinds of tumors and selected 656 datasets. The thresholds were set as the following: $p$ value (0.0001), fold change (2), gene rank (top 10\%), and data type (all).

2.2. cBioPortal with The Cancer Genome Atlas (TCGA) Dataset. The cBioPortal for Cancer Genomics [13, 14] (http://www.cbioportal.org) includes large scale of cancer genomic dataset that can be visualized and analyzed online. We performed the analyses on the dataset "TCGA, PanCancer Atlas," and the threshold was set as value \pm 2 .

2.3. Breast Cancer Gene-Expression Miner (bc-GenExMiner) v4.6. bc-GenExMiner v4.6 (http://bcgenex.ico.unicancer.fr/ BC-GEM/GEM-Accueil.php) [15-17] is a statistical mining tool of published annotated transcriptomic data. Statistical analyses are provided, including analyses of targeted expression, exhaustive expression, customized expression, targeted prognosis, exhaustive prognosis, molecular subtype prognosis, basal-like/TNBC prognosis, targeted gene correlations, exhaustive gene correlations, gene ontology, and gene correlations by chromosomal location. These data can be classified according to clinical and pathologic parameters.

2.4. ENCORI. The Encyclopedia of RNA Interactomes (ENCORI), (http://starbase.sysu.edu.cn/index.php), previously known as starBase v2.0 [18], is a public platform often used to analyze the interaction between mRNAs and noncoding RNAs among 23 species.

2.5. Cytoscope 3.8.2. The miRNA-mRNA network was drawn using Cytoscape 3.8.2 (http://www.cytoscape.org/) [19], which constructs complicated networks from original data.

2.6. TNMplot. TNMplot [20] (https://www.tnmplot.com/) is an online tool for the differential gene expression analysis among tumors.

2.7. UALCAN Analysis. UALCAN [21] (http://ualcan.path .uab.edu) uses related resources to analyze transcriptome data for 31 cancer types. It provides valuable data about genes or targets associated with clinical parameters.

2.8. GEO Datasets. GEO datasets (https://www.ncbi.nlm.nih .gov/gds/) contains original gene expression datasets, including raw data of sequencing, microarray, and platform information.

2.9. BCIP. BCIP (Breast Cancer Integrative Platform) [22] (http://www.omicsnet.org/bcancer/database) is a platform with gene expression, histopathological features, and clinical information of breast cancer samples.

2.10. Kaplan-Meier Plotter. Kaplan-Meier Plotter [23] (http://www.kmplot.com) is an online visualization tool for survival data for breast, lung, ovarian, liver, and gastric cancer. A $p$ value of $<0.05$ was considered statistically significant.

2.11. LinkedOmics Analysis. The LinkedOmics database [24] (http://www.linkedomics.org) is a publicly available platform with data for 32 TCGA cancer types. Differentially expressed

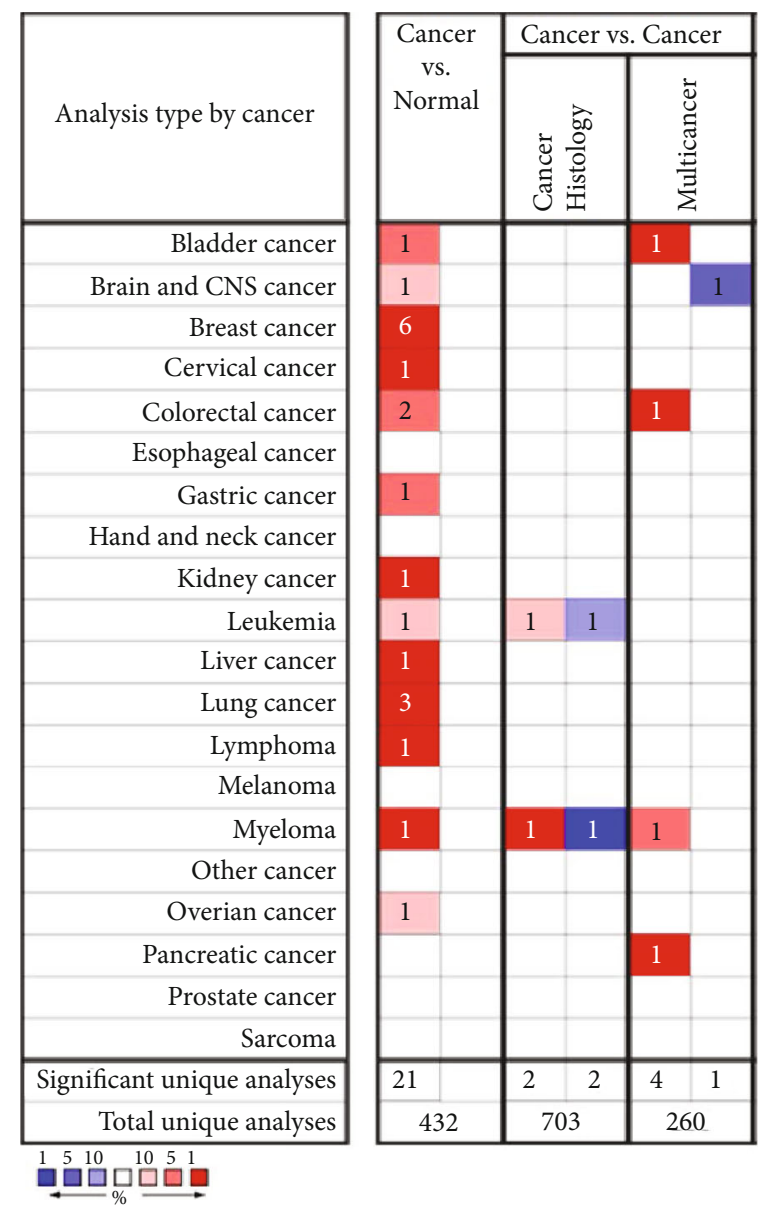

FIgURe 1: The transcription level of the FLAD1 in 20 types of human cancers in Oncomine.

genes correlated with FLAD1 in the TCGA breast invasive carcinoma cohort were identified. Results were analyzed using Spearman's correlation coefficients.

\section{Results}

3.1. FLAD1 Overexpression in Pan-Cancer Analysis. We first analyzed the FLAD1 expression in human cancers and found that it was overexpressed in six breast cancer datasets in the Oncomine database (Figure 1). We also found some evidence for the abnormal expression of FLAD1 in various cancers in ENCORI [18]. A significant difference in the FLAD1 expression between cancer samples and normal tissue samples was identified for eight cancer types (Figure 2, $p<0.001$ ), including kidney renal papillary cell carcinoma, head-neck squamous cell carcinoma, esophageal cancers, colon adenocarcinoma, cholangiocarcinoma, breast invasive carcinoma, bladder urothelial carcinoma, and liver hepatocellular carcinoma. TNMplot [20] was further used for pan-cancer analysis of FLAD1, including 56,938 samples showing that FLAD1 is highly expressed in acute myeloid leukemi, bladder cancer, breast cancer, colon cancer, esophageal cancer, liver cancer, lung adenocarcinoma, lung squamous cell cancer, ovarian cancer, pancreatic cancer, rectum cancer, kidney renal 


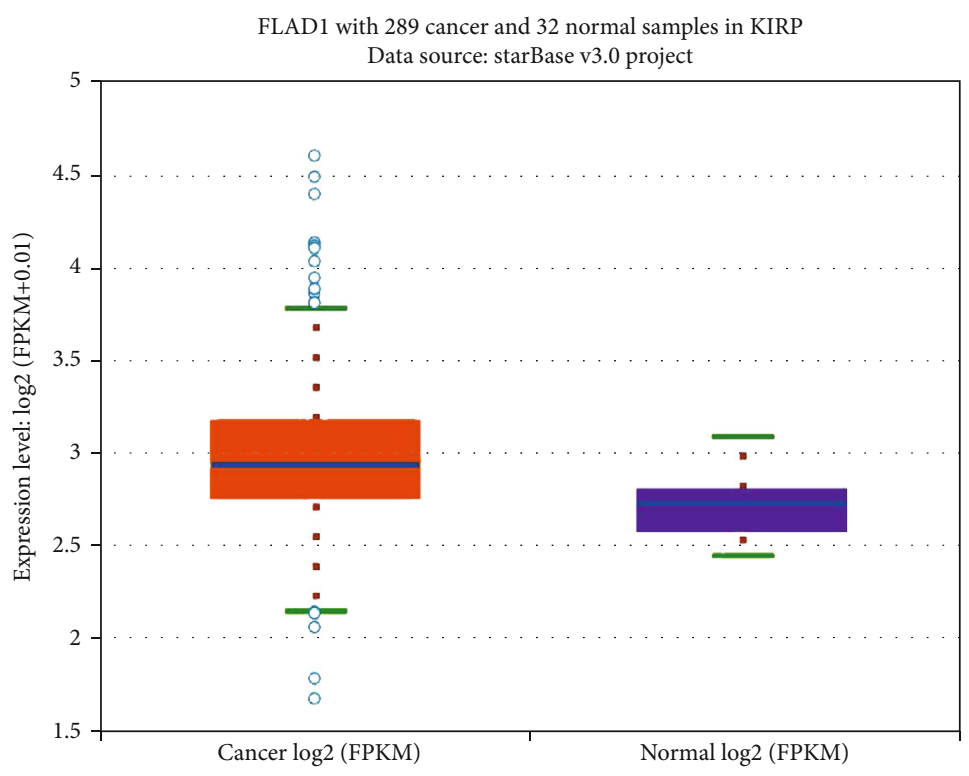

(a)

FLAD1 with 502 cancer and 44 normal samples in HNSC

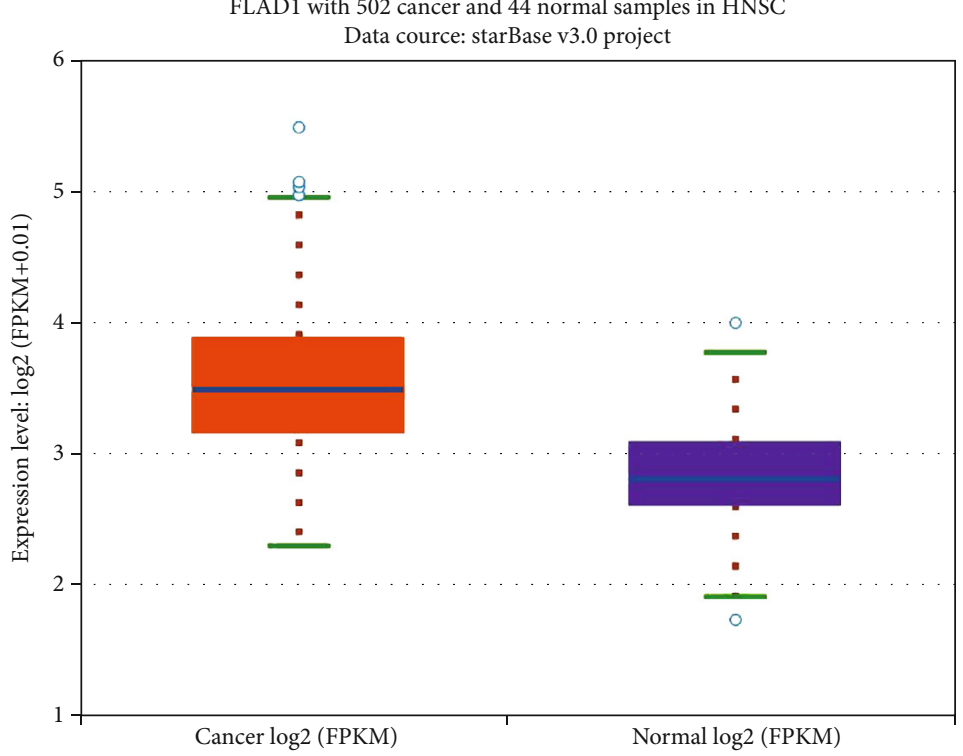

(b)

Figure 2: Continued. 


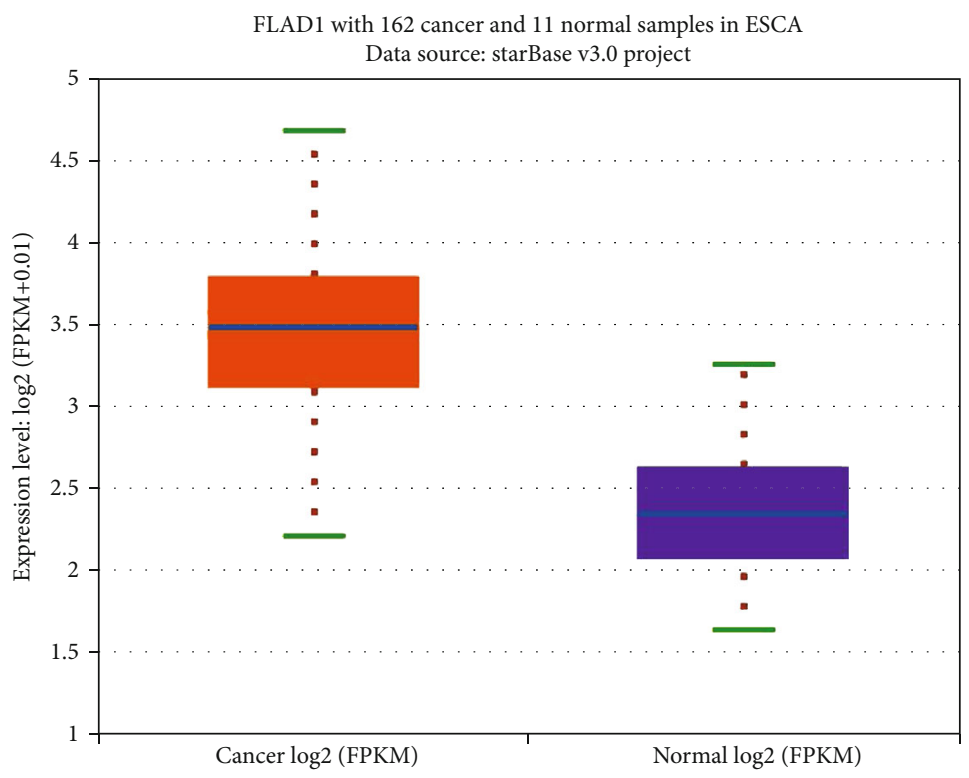

(c)

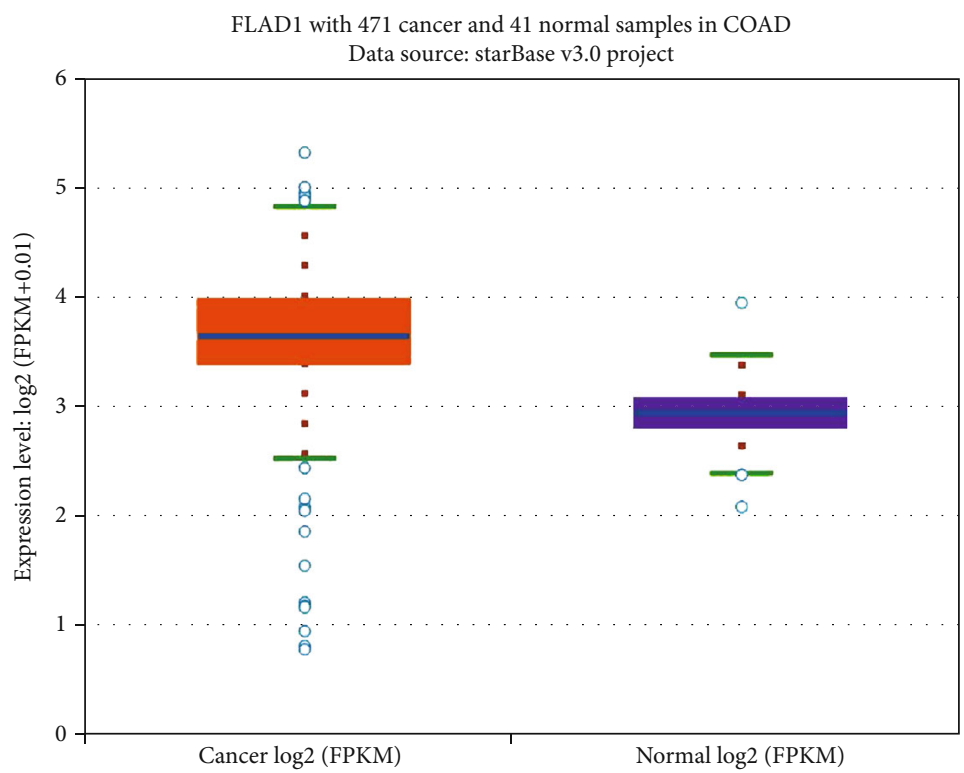

(d)

Figure 2: Continued. 
FLAD1 with 36 cancer and 9 normal samples in CHOL Data source: starBase v3.0 project

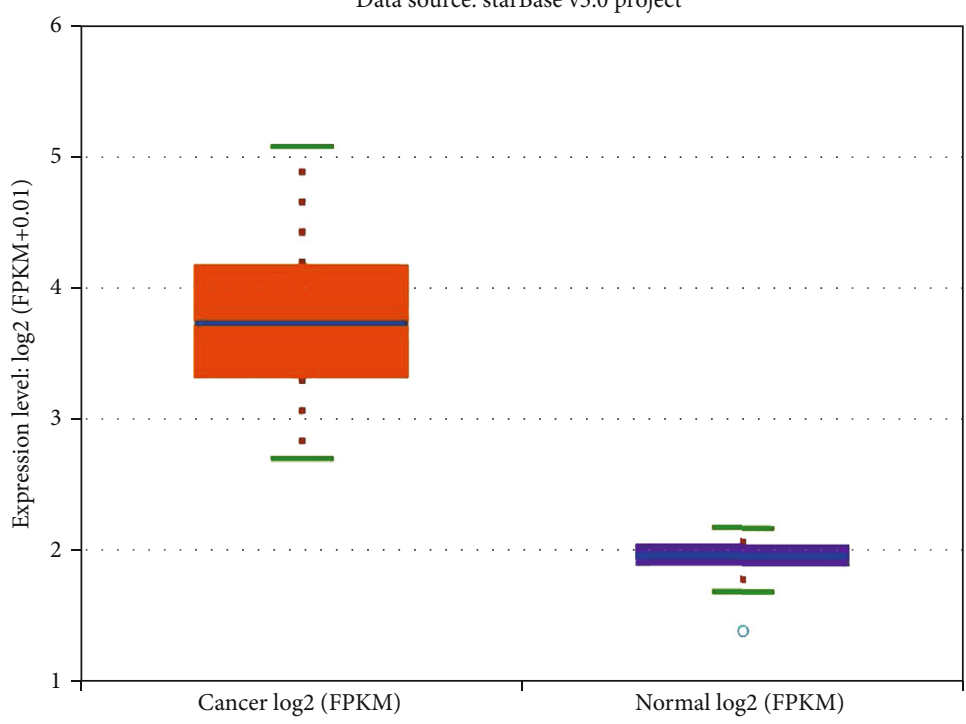

(e)

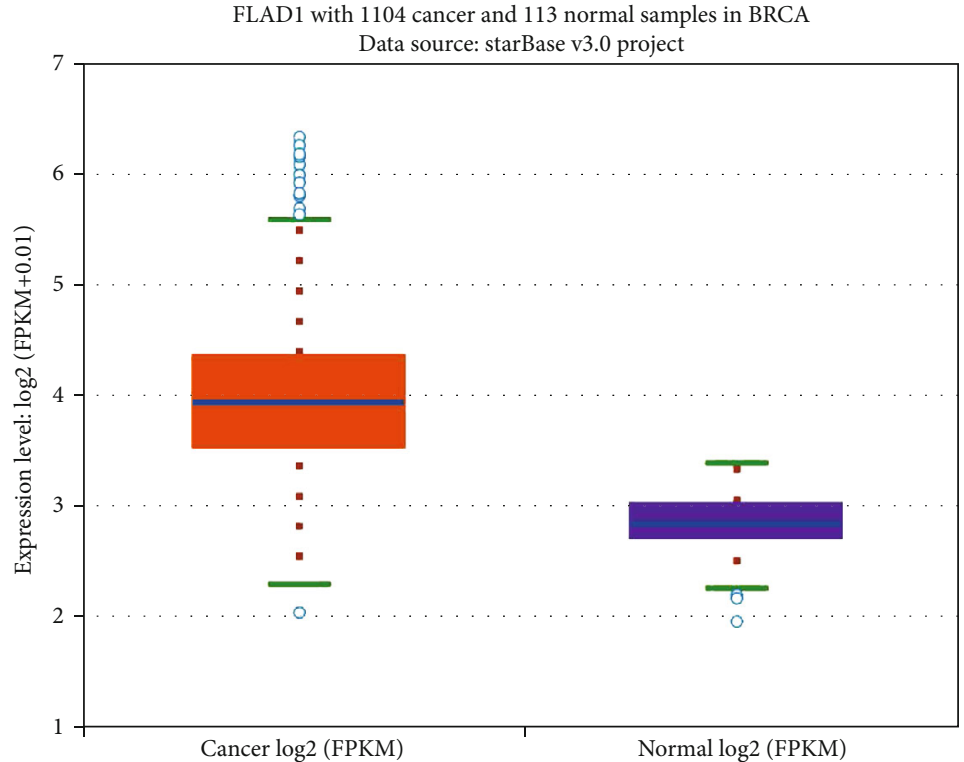

(f)

FIGURE 2: Continued. 


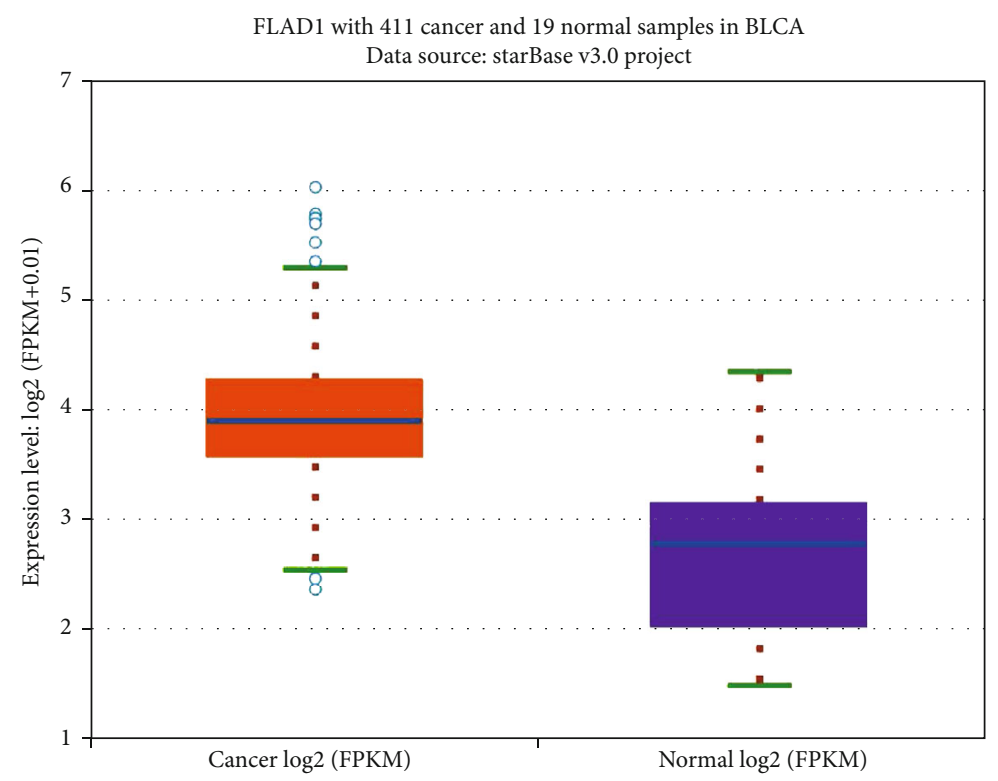

(g)

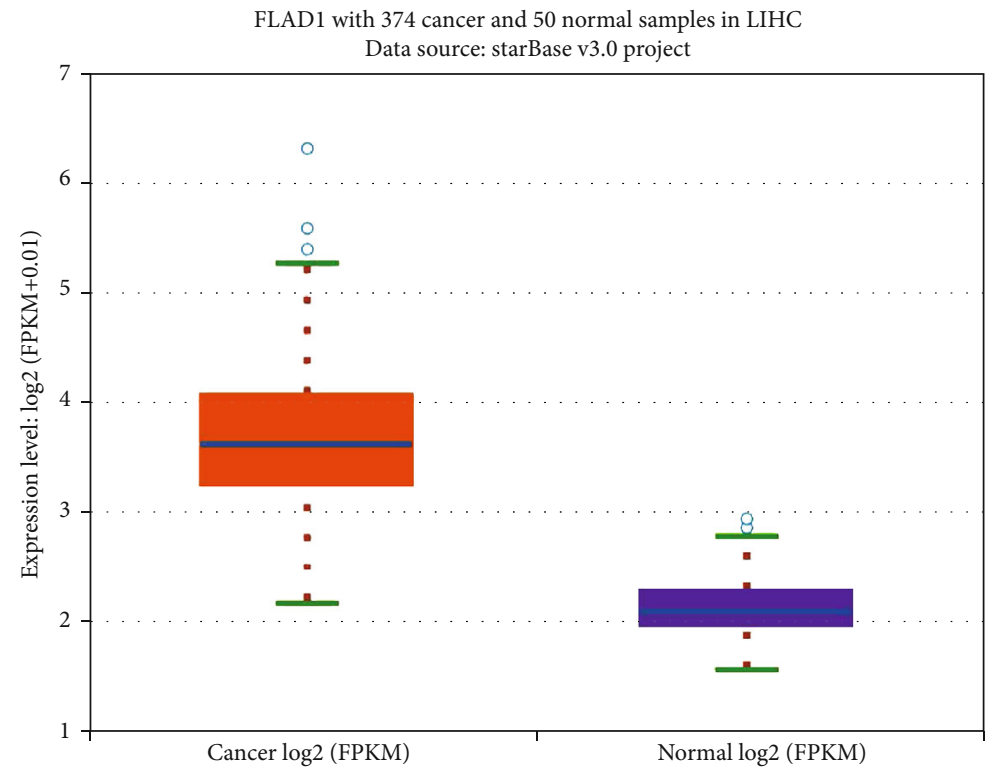

(h)

FIGURE 2: The transcription level of FLAD1 in various cancer types in ENCORI: (a) kidney renal papillary cell carcinoma (KIRP), (b) headneck squamous cell carcinoma (HNSC), (c) esophageal cancers (ESCA), (d) colon adenocarcinoma (COAD), (e) cholangiocarcinoma (CHOL), (f) breast invasive carcinoma (BRCA), (g) bladder urothelial carcinoma (BLCA), and (h) liver hepatocellular carcinoma (LIHC). $p<0.001$ in all figures.

papillary cell carcinoma, skin cancer, stomach cancer, testicular cancer, uterine carcinosarcoma, and uterine corpus endometrial carcinoma (Figure 3, $p<0.001$ ).

\subsection{Frequency and Type of FLAD1 Alterations in Breast} Cancer. We used cBioPortal to determine the type and frequency of FLAD1 alterations in 106 of 996 patients with breast cancer. Only 10 cases were with mutation while 5 cases had missense mutations, 4 cases had truncation mutations, and 1 had SV/Fusion. Thus, amplification may be the most common type of FLAD1 alteration in breast cancer.
3.3. Diagnostic Value and Related Clinical Features of the FLAD1 Expression in Breast Cancer. We then analyzed the FLAD1 expression in three GEO datasets (GSE10797 [25], GSE22820 [26], GSE54002 [27]) and in BCIP ("Metabric," [28] “TCGA_Agilent," “GSE5364_GPL96” [29]). These results confirmed that the expressions levels of FLAD1 in breast cancer groups are higher than that in normal tissue groups (Figures 4 and 5).

The analysis using bc-GenExMiner showed that the ERpositive and PR-positive groups had lower FLAD1 expression $(p<0.001)$ in line with a previous study [11]. However, the HER2-positive groups showed higher FLAD1 expression 


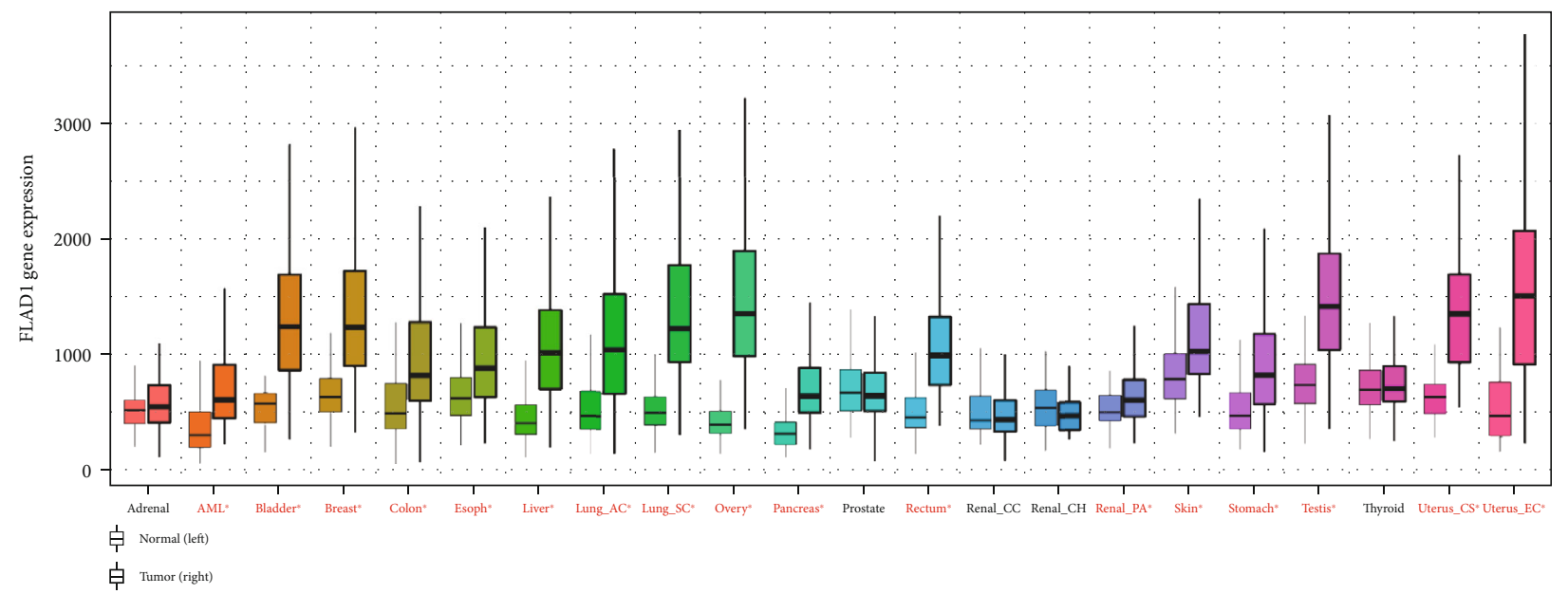

FIGURE 3: Boxplots of the FLAD1 gene expression in 22 tumor types. Significant differences by a Mann-Whitney $U$ test are marked with asterisk and red color $\left({ }^{*} p<0.01\right)$. Note: AML: acute myeloid leukemia.

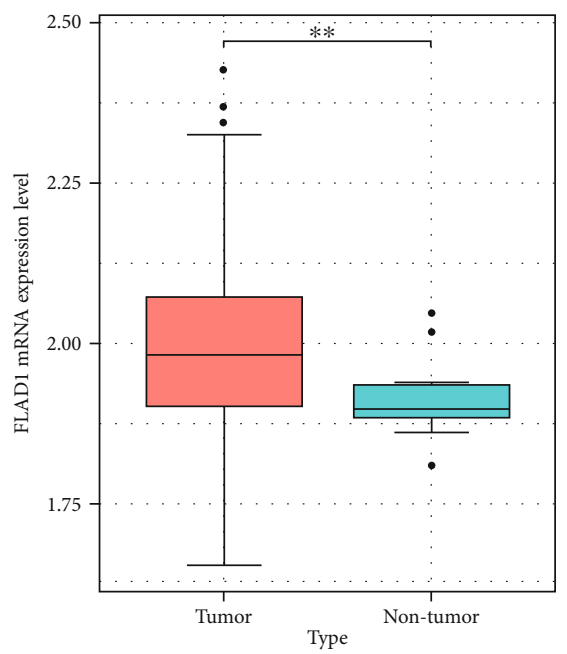

(a)

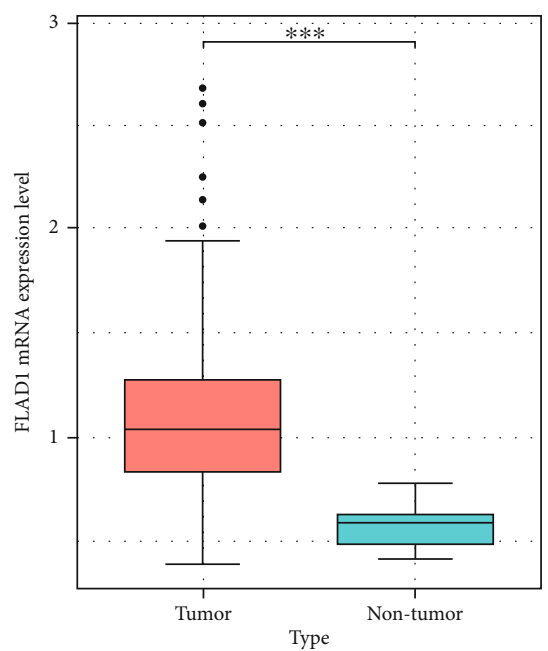

(b)

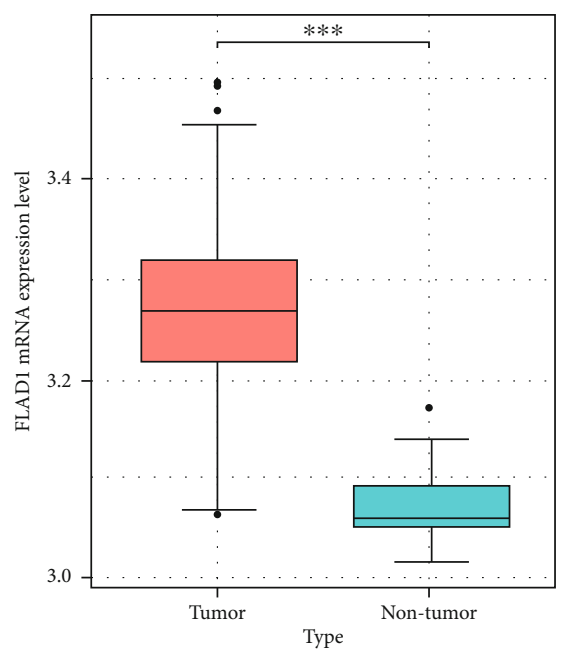

(c)

FIGURE 4: The FLAD1 expression in breast cancers samples and normal controls from GEO. (a) GSE10797, $p=0.0067$. (b) GSE22820, $p<$ 0.001. (c) GSE54002, $p<0.001 .{ }^{*} p<0.05 ;{ }^{* *} p<0.01 ;{ }^{* * *} p<0.001$. 


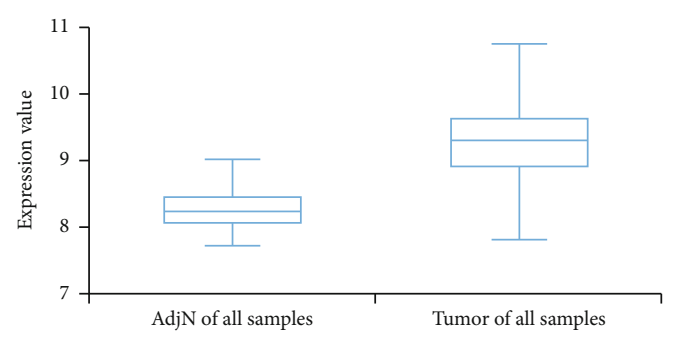

(a)

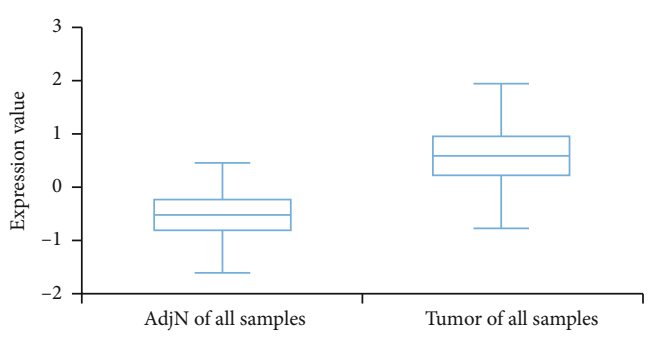

(b)

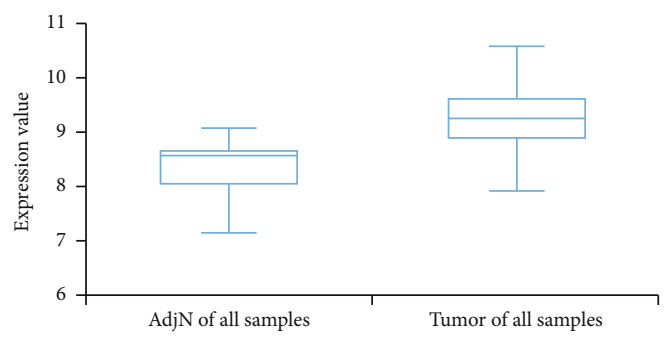

(c)

Figure 5: The FLAD1 expression in breast cancers samples and normal controls from BCIP. (a) Dataset "Metabric", $p<0.001$. (b) Dataset “TCGA_Agilent”, $p<0.001$. (c) Dataset “GSE5364_GPL96”, $p=0.008$.

(Figure 6(a), $p<0.001$ ). Nodal status (Figure 6(b) $p=0.0011$ ) and age (Figure 6(c), $p<0.001$ ) were also related to the FLAD1 expression. In addition, the p53 mutated group showed higher FLAD1 expression (Figure 6(d), $p<0.001$ ). The basal-like group showed higher FLAD1 expression levels than those found for samples with a nonbasal-like status (Figure $6(\mathrm{e}), p<0.001)$. Similarly, the triple-negative group showed higher mRNA expression levels than those in the nontriple-negative group. Moreover, the expression level of FLAD1 sequentially increased in advanced stages based on the NPI (Figure 6(f), $p<0.001$ ).

UALCAN was also used to reveal the clinical parameters related to FLAD1 in breast invasive carcinoma. The overall FLAD1 mRNA expression level was significantly $(p<0.001)$ higher in the breast cancer group than in the healthy donors (Figure 7(a); median transcripts per million (TPM) of 58.593 and 29.68, respectively). There was no significant difference in the FLAD1 levels according to gender (Figure 7(b), median TPM 58.821 for men and 58.519 for women, respectively; $p=0.818$ ); although, significant differences were found for age (Figure $7(\mathrm{c})$ ); the median TPM for patients who were 21-40 years old (median TPM $=66.653$ ) was higher than those of other age groups: $41-60$ years (median TPM $=60.918, p=0.016$ ), $61-80$ years (median TPM $=54.837, p=0.002)$, and $81-100$ years (median TPM $=55.355, p=0.003$ ). Significant differences were also found with respect to race, with significantly higher expression levels being observed in African-American patients (median TPM $=68.061$ ) than in Caucasian (median TPM $=56.558$ ) and Asian (median TPM $=60.17$ ) patients $(p<0.001$; Figure $7(\mathrm{~d})$ ). There were no differences observed in the comparisons of the patients from the Caucasian vs. Asian $(p=0.078)$ or African-American vs. Asian $(p=0.332)$ groups. The FLAD1 expression level was higher for all stages compared with the normal group. However, a significant difference $(p=0.003)$ between the stages was only found for stage 1 (median TPM $=52.445)$ and stage 2 (median TPM $=60.417$ ), with no significant differences for the other comparisons: stage 1 vs. stage 3 (median TPM $=56.524$ ), $p=0.135$; stage 1 vs. stage 4 (median TPM $=66.849$ ), $p=0.064$; stage 2 vs. stage $3, p=$ 0.255 , stage 2 vs. stage $4, p=0.492$; and stage 3 vs. stage 4 , $p=0.320$ (Figure $7(\mathrm{e})$ ). In addition, the FLAD1 expression levels in the luminal subclass (median TPM $=56.156$ ) were also significantly lower than those in the HER2-positive (median TPM $=67.728$ ) and triple-negative (median TPM $=76.715$ ) groups (Figure $7(\mathrm{f}), p=0.003$ and $p<0.001$, respectively), whereas no significant difference was found between the FLAD1 expression levels in the HER2-positive and triple-negative groups $(p=0.232)$.

3.4. Prognostic Value of the FLAD1 Expression in Breast Cancer and Other Cancers. We analyzed the survival data from the BCIP, which showed that the low expression of FLAD1 is associated with longer overall survival (OS) and disease-specific survival (Figure 8).

We also analyzed the relationships between the FLAD1 expression and OS in a number of different kinds of cancers and found that the FLAD1 overexpression was related to a poorer OS in five types of cancers: kidney renal clear cell carcinoma, kidney renal papillary cell carcinoma, liver hepatocellular carcinoma, sarcoma, and thymoma (Figure 9).

3.5. FLAD1-Related miRNA Network and Prognostic Value. We used ENCORI to analyze the mRNA-miRNA interactions and then used cystoscope to visualize the network. The related miRNAs were identified included hsa-miR-1283p, hsa-miR-137, hsa-miR-299-5p, hsa-miR-3622a-5p, hsamiR-486-5p, and hsa-miR-154-5p (Figure 10).

We then performed the survival analysis for each miRNA and found that longer survival time is positively correlated with hsa-miR-299-5p, hsa-miR-154, hsa-miR-299-3p, hsa- 

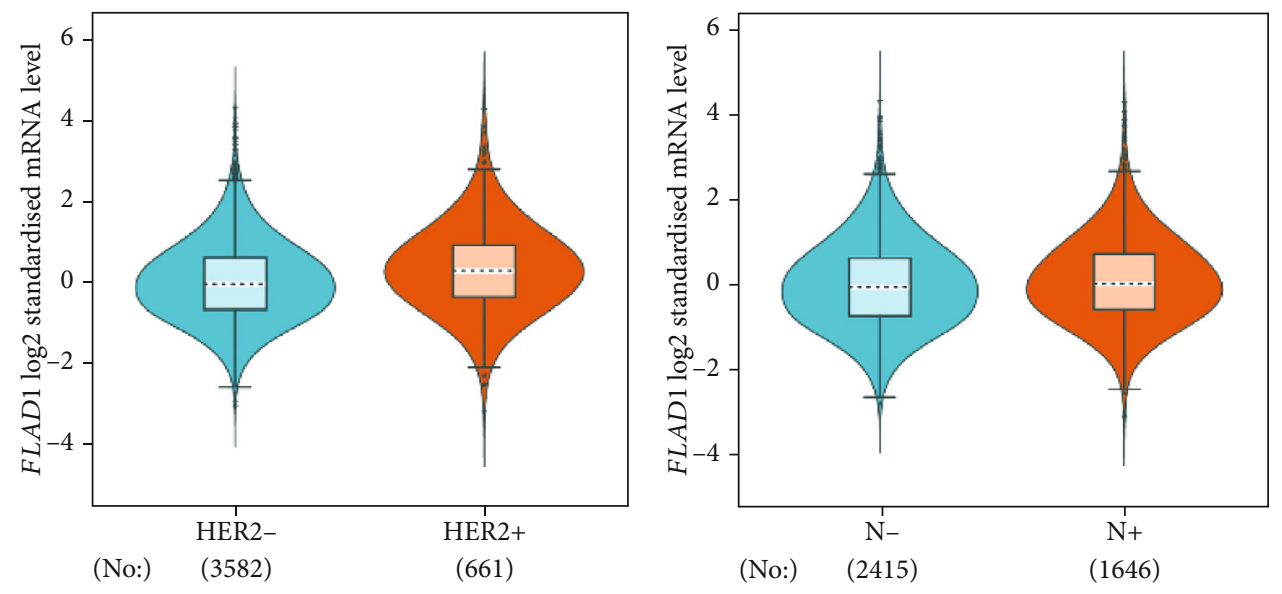

$p<0.0001$
$<\square$

$$
p<0.0011
$$

(a)

(b)
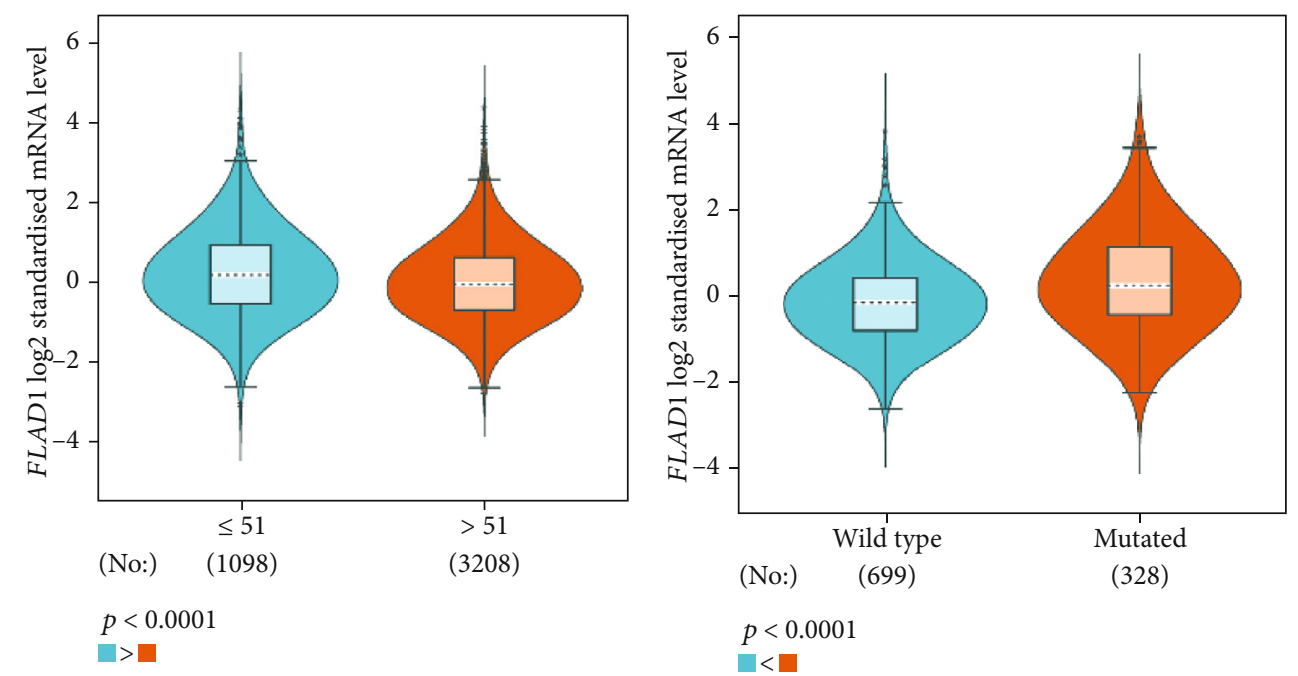

$p<0.0001$

(c)

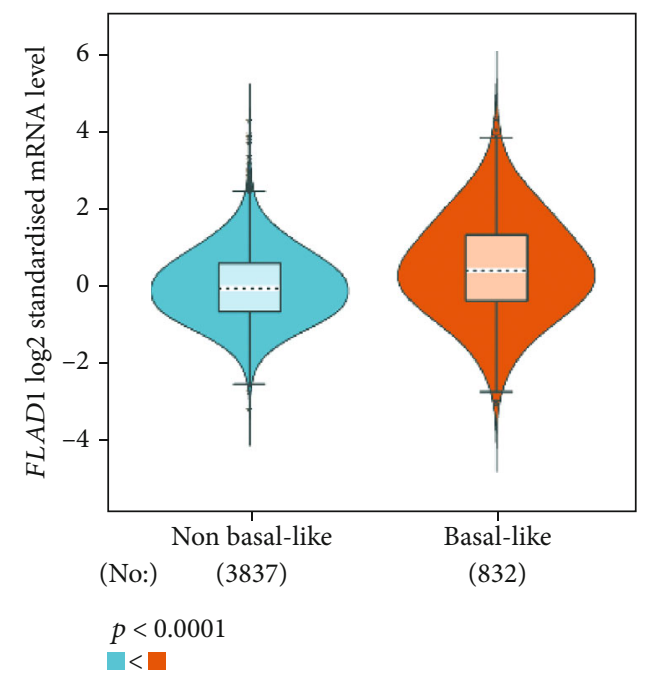

(e)

(d)

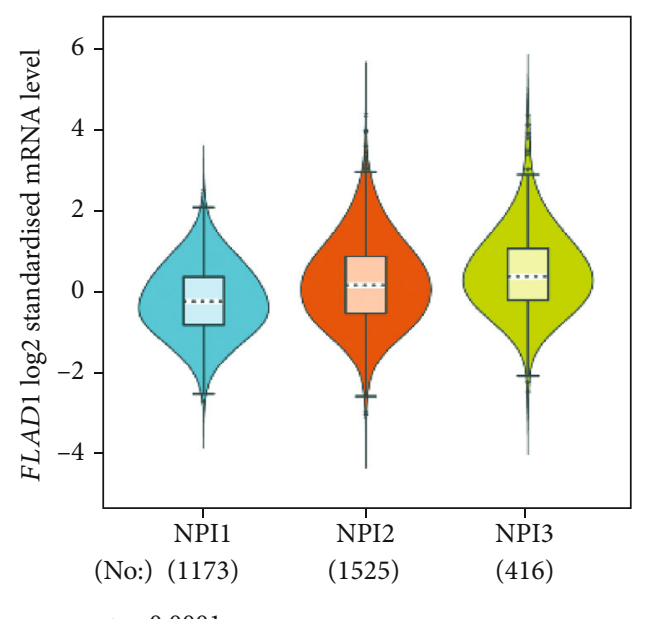

$p<0.0001$

(f)

FIGURE 6: Violin plots showed the FLAD1 mRNA expression in subgroups of patients with breast cancer (bc-GenExMiner). (a) Expressions between HER2 (-) and HER2 (+). (b) Expression related to nodal status. (c) Expressions between age $\leq 51$ and age $>51$. (d) Expressions between p53 wild type and mutation. (e) Expressions between basal-like and nonbasal-like status. (f) Expression among NPI 1, 2 , and 3. 
Expression of FLAD1 in BRCA based on sample types

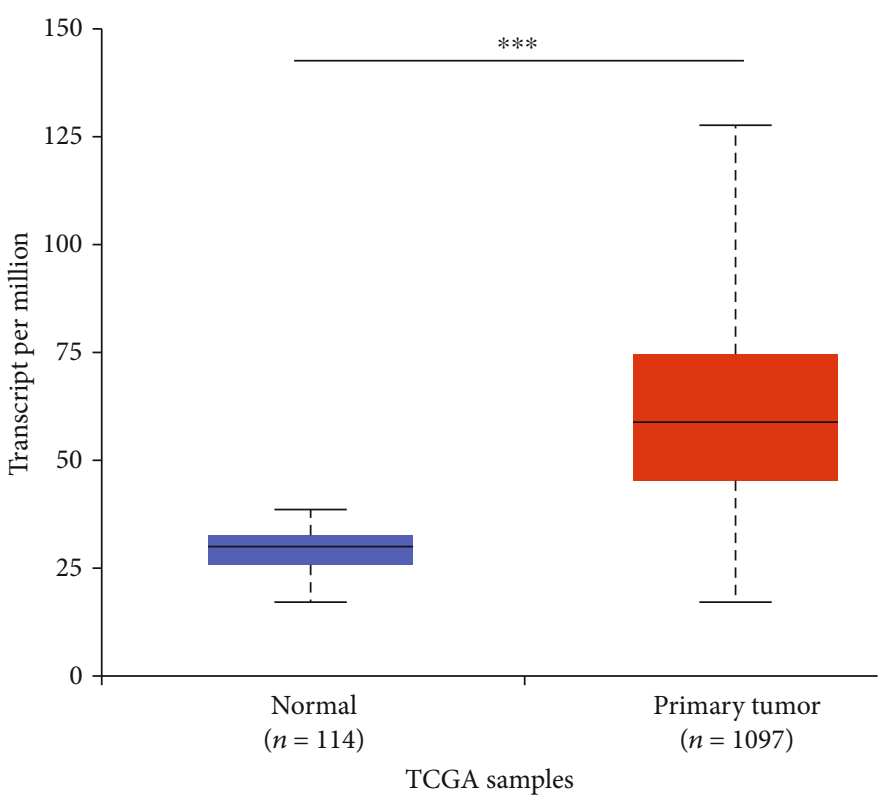

(a)

Expression of FLAD1 in BRCA based on patient's gender

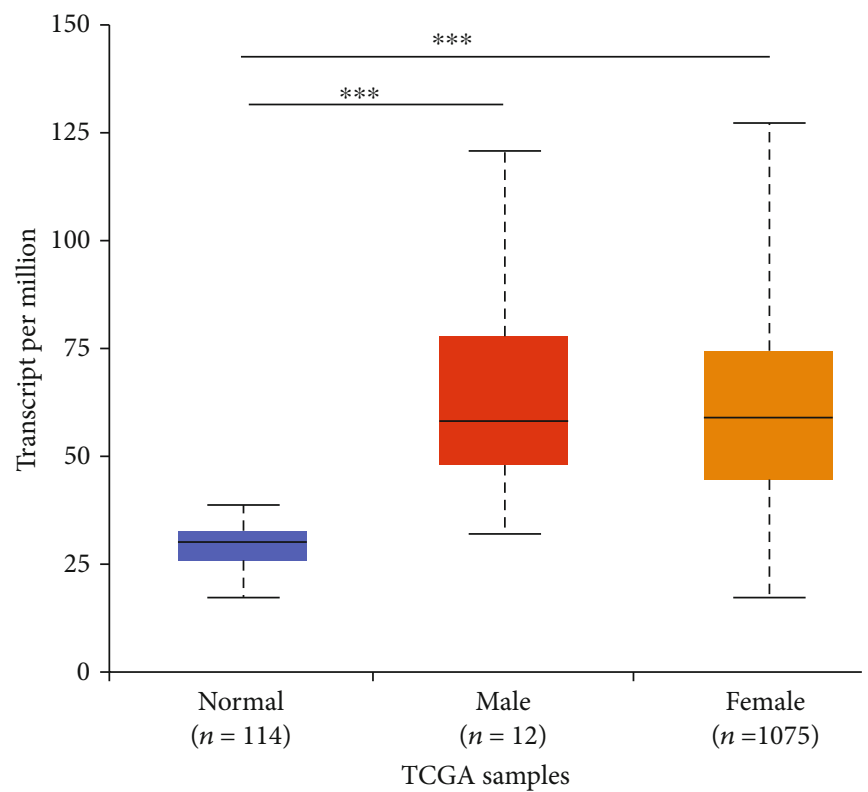

(b)

FIGURe 7: Continued. 


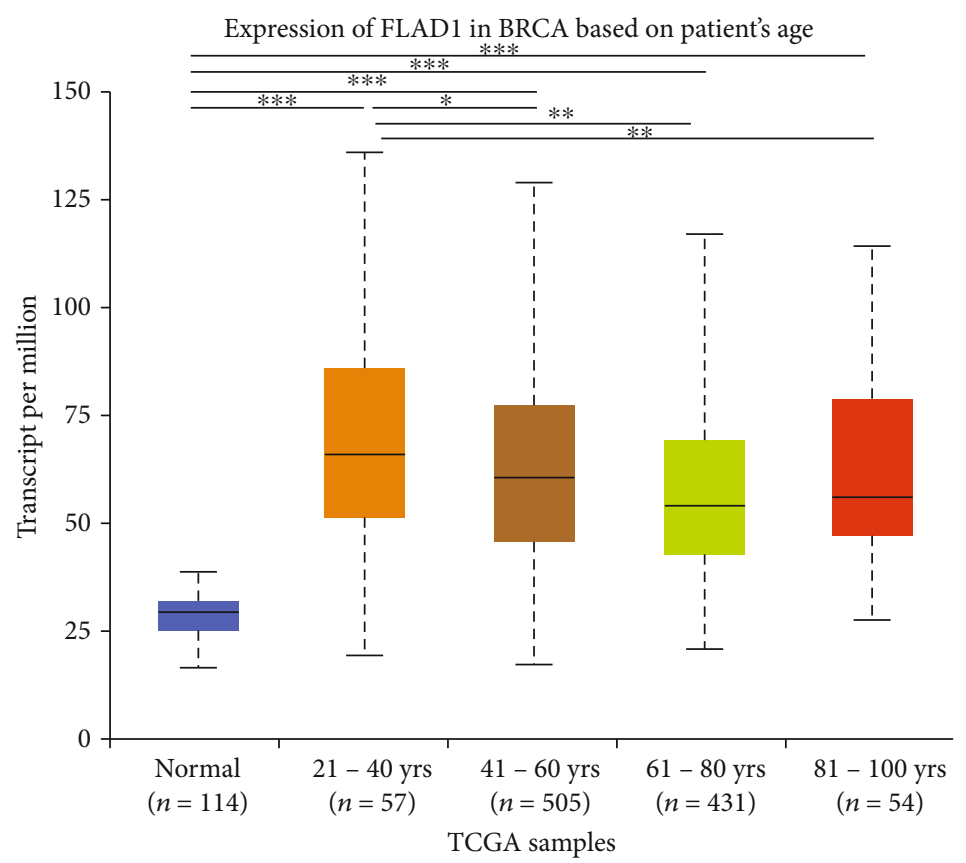

(c)

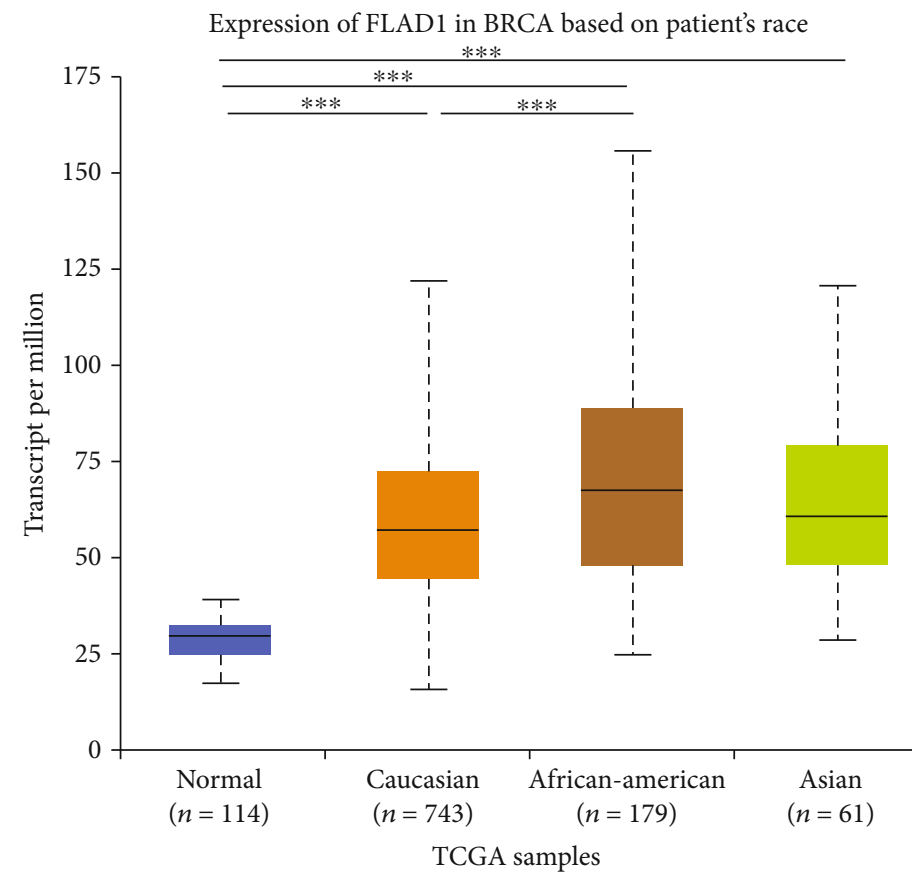

(d)

FIgure 7: Continued. 
Expression of FLAD1 in BRCA based on individual cancer stages

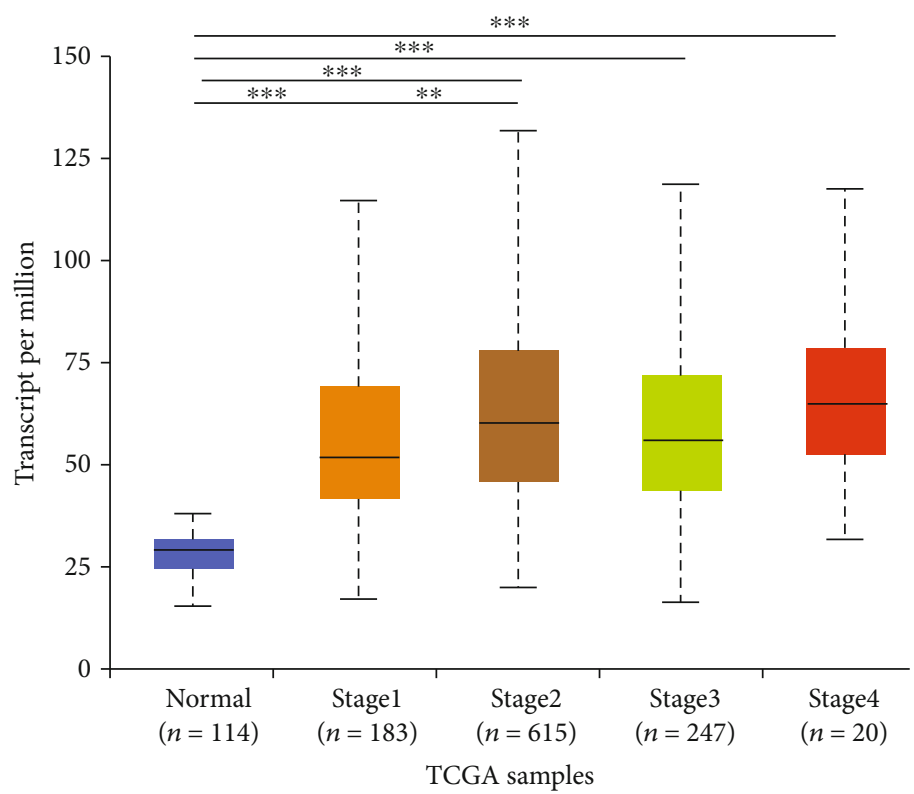

(e)

Expression of FLAD1 in BRCA based on breast cancer subclasses

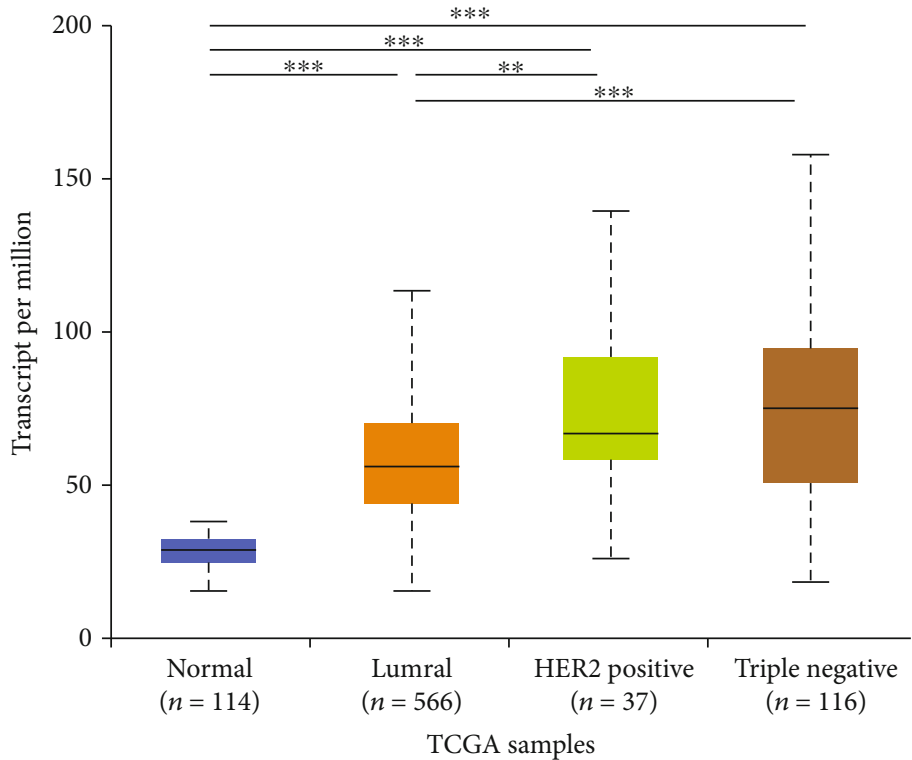

(f)

FIGURE 7: Boxplots showed FLAD1 transcription in subgroups of patients with breast invasive carcinoma (UALCAN). (a) Comparison between the normal group and cancer group. (b) Comparison between the normal group and different genders in patients' group (male and female). (c) Comparison between the normal group and different ages in patients' group (21-40, 41-60, 61-80, or 81-100 years). (d) Comparison between the normal group and different races in patients' group (Caucasian, African-American or Asian). (e) Comparison between the normal group and different stages in patients' group (stages 1, 2, 3, or 4). (f) Comparison between the normal individuals and different subclasses in patients' group (luminal, HER2-positive, and triple-negative). Data are mean $\pm S E$. ${ }^{*} p<0.05$; ${ }^{* *} p<0.01 ;{ }^{* * *} p<0.001$.

miR-31, hsa-miR-328, hsa-miR-654-5p, and hsa-miR-543 (Figure S1, $p<0.05$ ). In addition, we found that the low expression of some miRNAs was associated with a higher survival rate, including hsa-miR-3622a, hsa-miR-1343, hsamiR-24, hsa-miR-541, hsa-miR-3918, hsa-miR-224, hsamiR-4731, hsa-miR-4726, hsa-miR-378 g, hsa-miR-4739, 


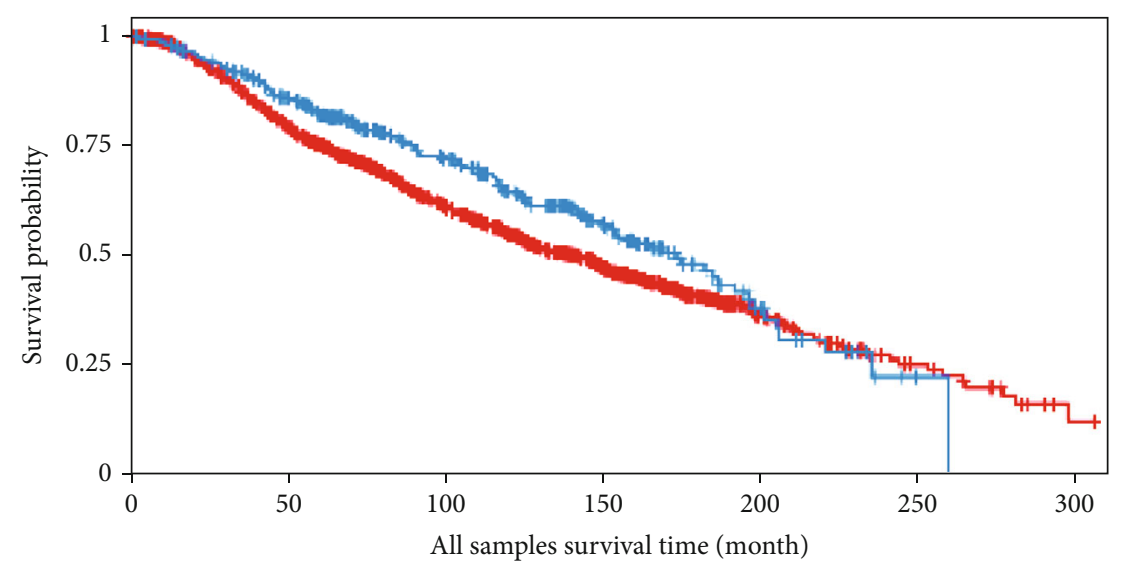

— High expression group

_ Low expression group

(a)

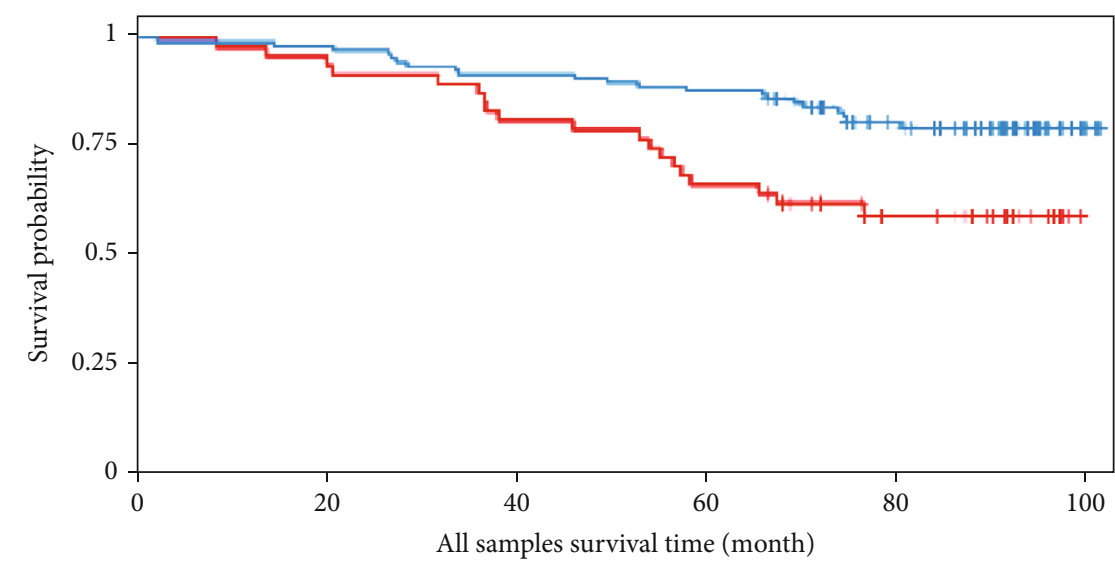

- High expression group

— Low expression group

(b)

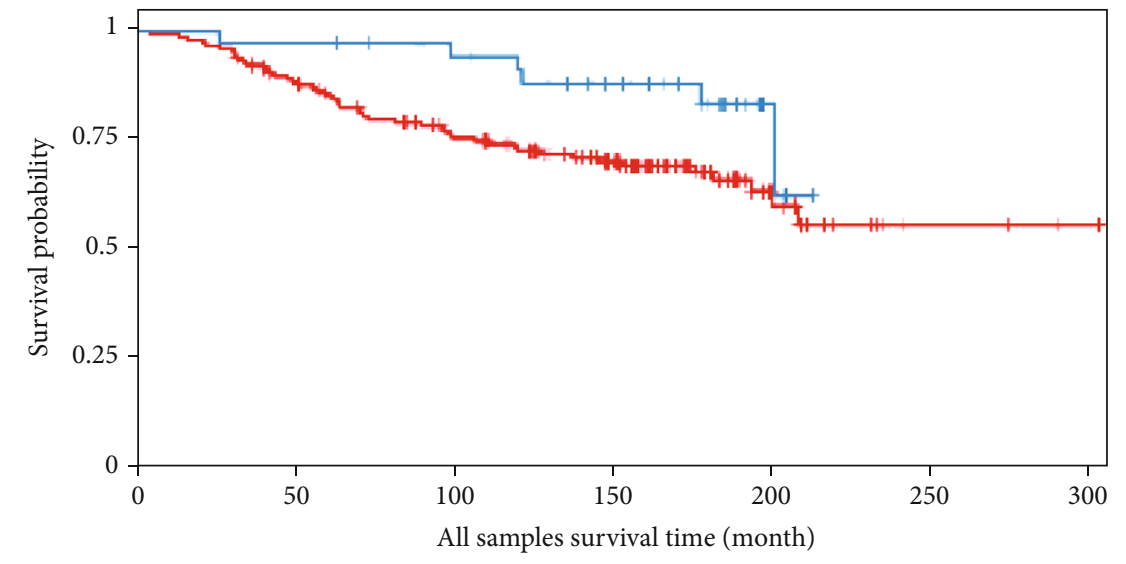

— High expression group

_ Low expression group

(c)

Figure 8: Continued. 


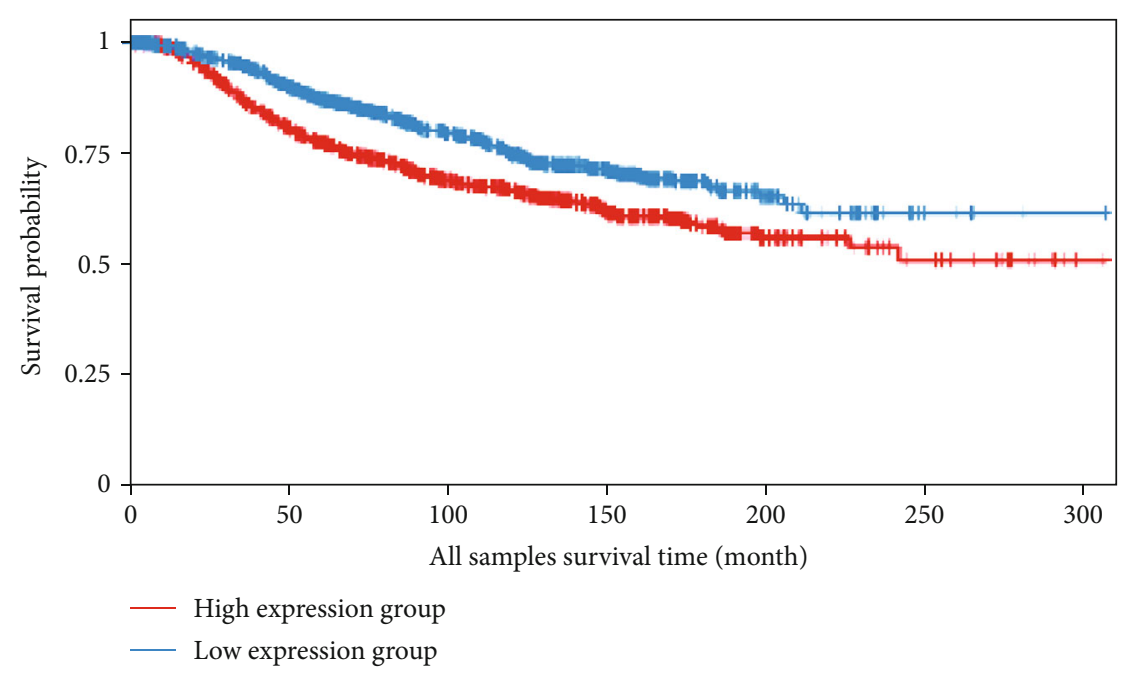

(d)

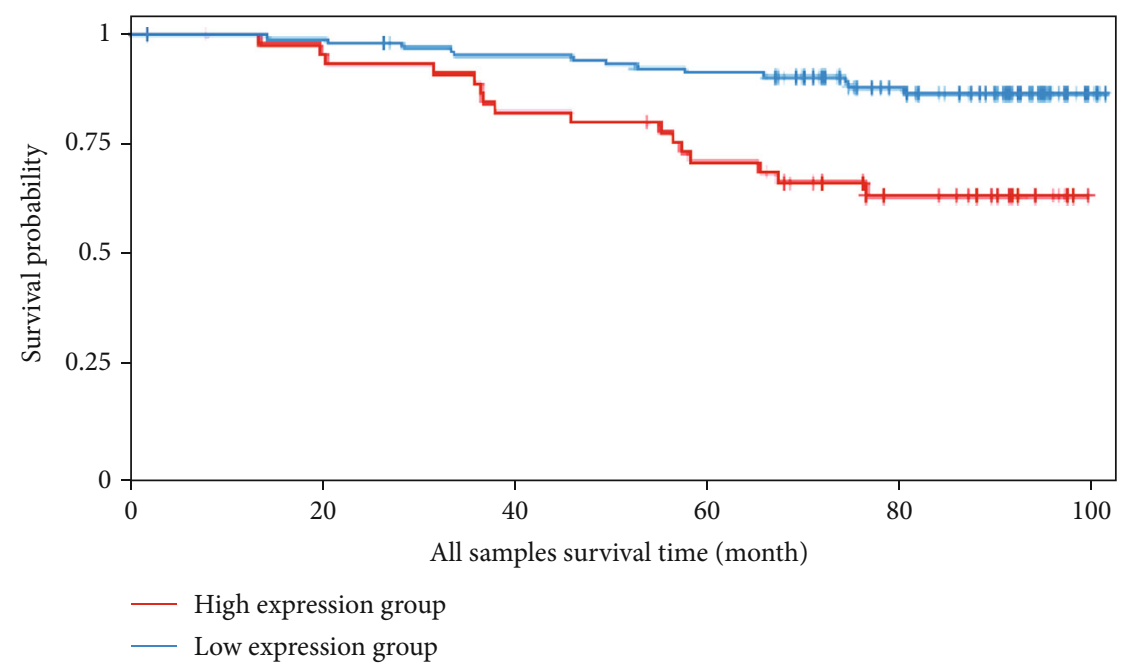

(e)

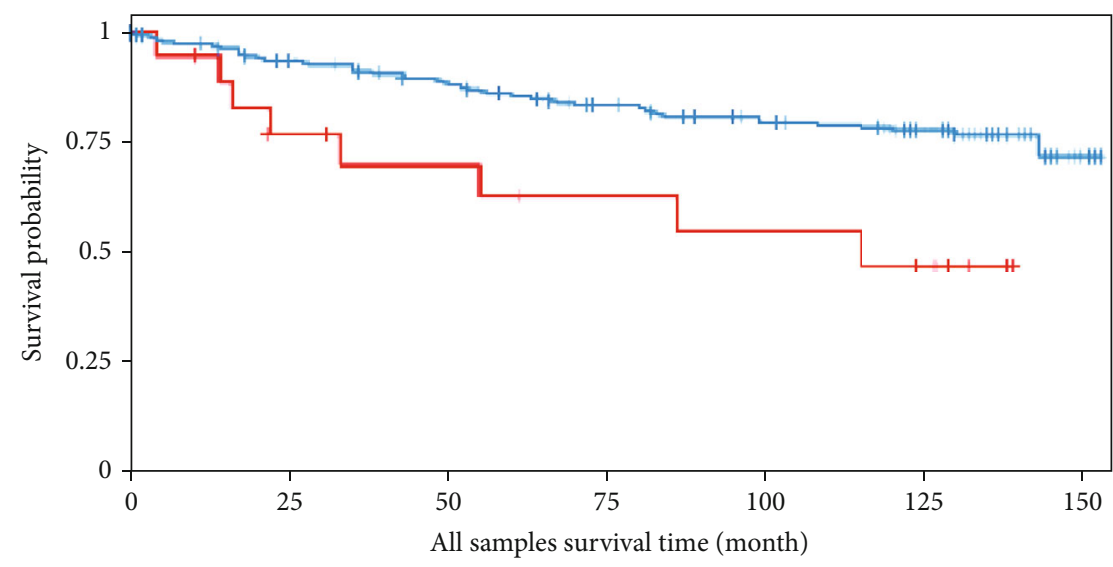

- High expression group

- Low expression group

(f)

Figure 8: Overall survival (OS) and disease-specific survival (DS) between the high and low expression of the FLAD1 group. (a) OS from dataset "Metabric" [28], $p=0.003$. (b) OS from dataset "GSE1456_GPL96" [30], $p=0.004$. (c) OS from dataset "GSE7390_GPL96" [31], $p=0.046$. (d) DS from dataset "Metabric", $p<0.001$. (e) DS from dataset "GSE1456_GPL96", $p<0.001$. (f) DS from dataset “GSE3494_GPL96" [32], $p=0.004$. 


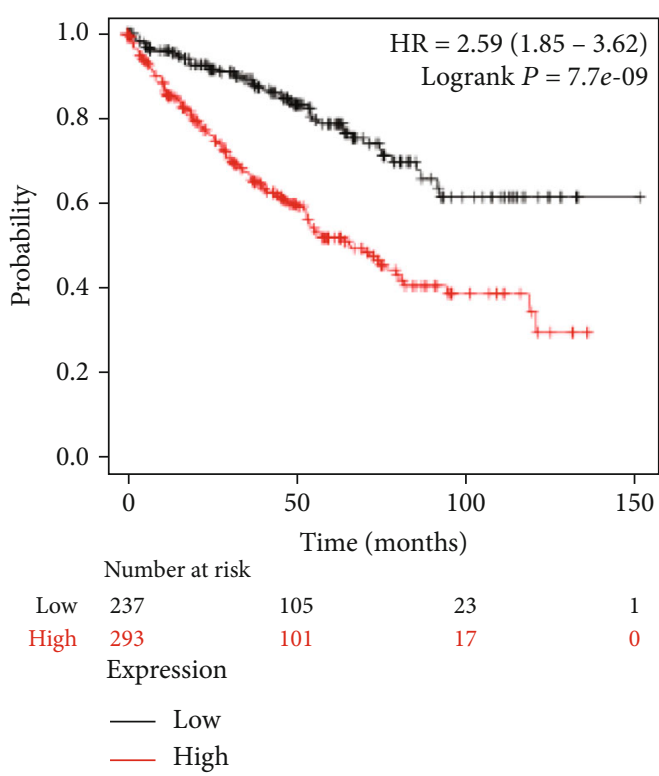

(a)

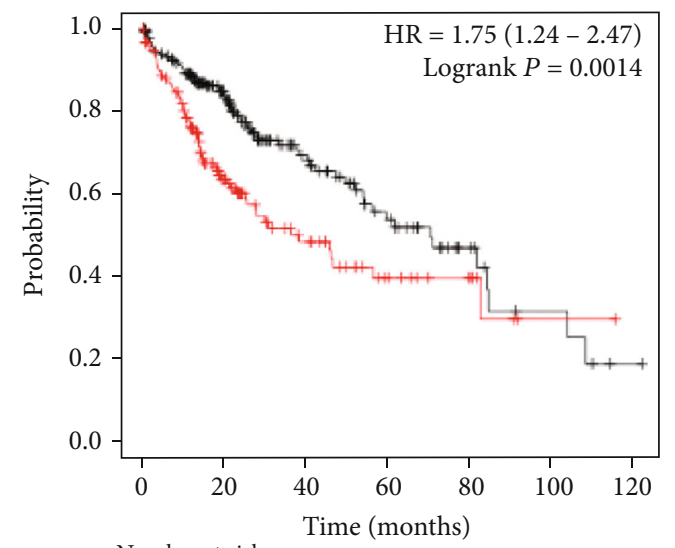

Number at risk

$\begin{array}{cccccccc}\text { Low } & 211 & 120 & 55 & 29 & 12 & 5 & 1 \\ \text { High } & 159 & 62 & 29 & 13 & 7 & 1 & 0\end{array}$

Expression

— Low

_ High

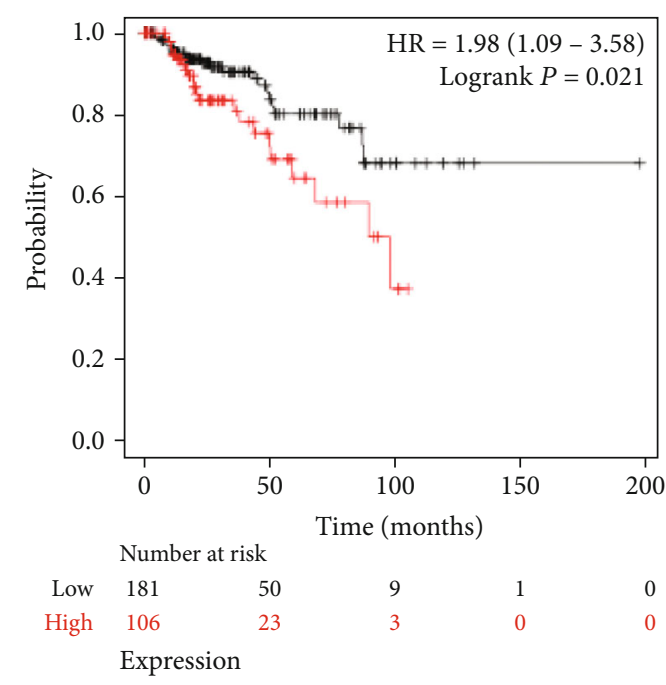

- Low

_ High

(b)

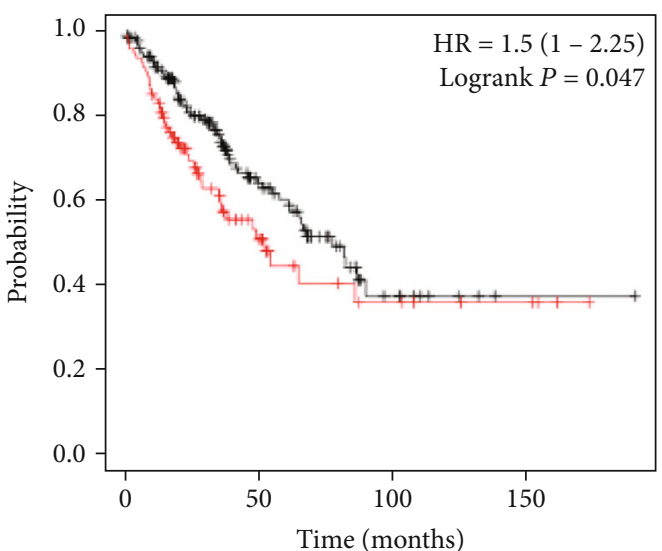

Number at rsik

$\begin{array}{ccccc}\text { Low } & 166 & 52 & 9 & 1 \\ \text { High } & 93 & 21 & 7 & 4 \\ & \text { Expression } & & & \end{array}$

— Low

— High

(c)

(d)

Figure 9: Continued. 


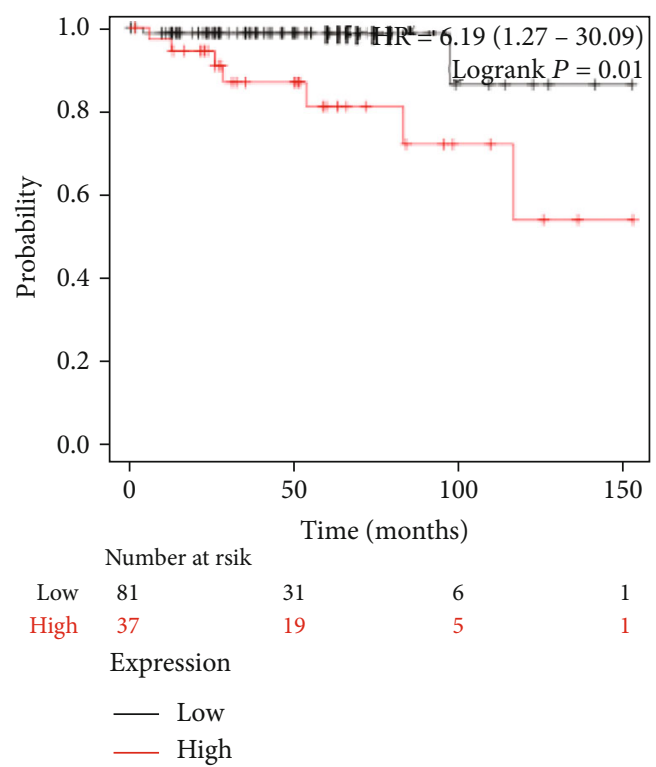

(e)

FIGURE 9: Survival analyses of the FLAD1 expression in cancers: (a) kidney renal clear cell carcinoma, (b) kidney renal papillary cell carcinoma, (c) liver hepatocellular carcinoma, (d) sarcoma, and (e) thymoma.

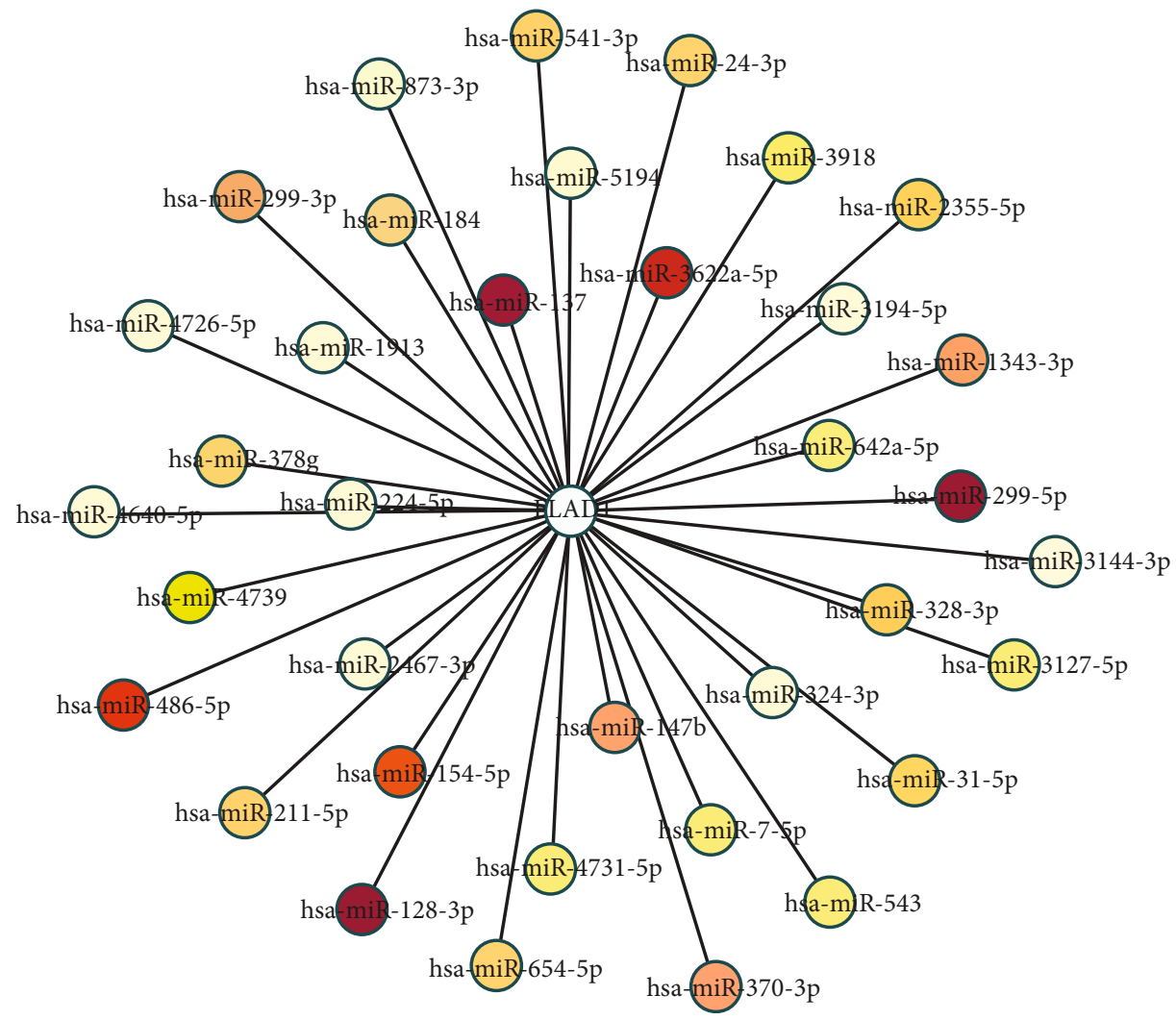

FIGURE 10: FLAD1-related miRNAs. Darker color (red) represents more experimental evidence.

hsa-miR-7, hsa-miR-4640, hsa-miR-1913, hsa-miR-2467, hsa-miR-3144, and hsa-miR-5194 (Figure S2).

3.6. Genes Correlated with FLAD1 in Breast Invasive Carcinoma. We used LinkedOmics to analyze proteomic data for patients with breast invasive carcinoma and found 1861 genes (dark red dots in Figure 11) showing significant positive correlations with FLAD1 and 1870 genes (dark green dots) showing significant negative correlations, as shown in a volcano plot (Figure $11(\mathrm{a})$ ) (false discovery rate $[\mathrm{FDR}]<$ 


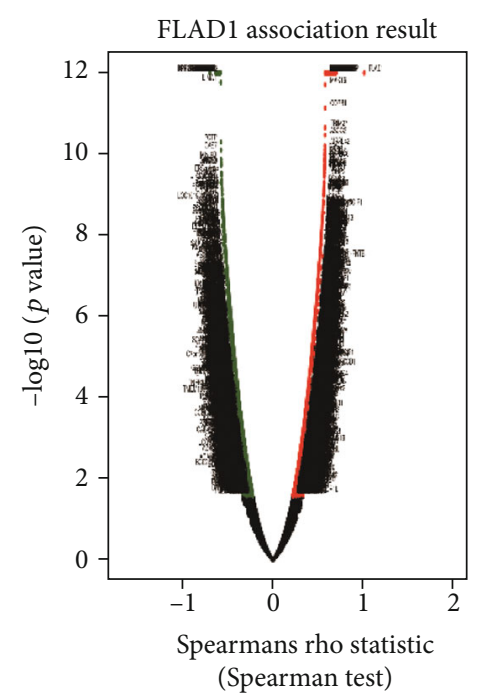

(a)

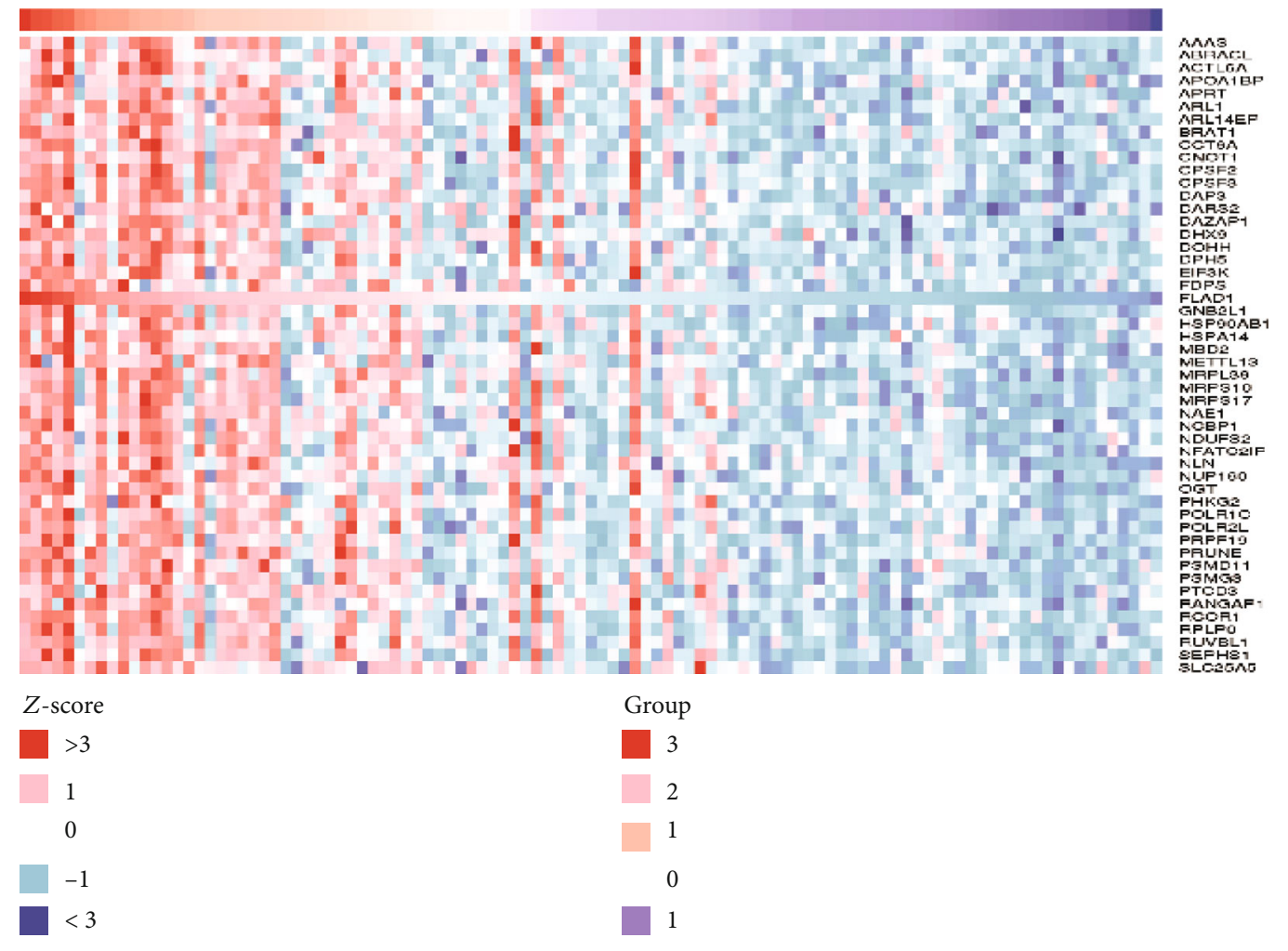

(b)

Figure 11: Continued. 


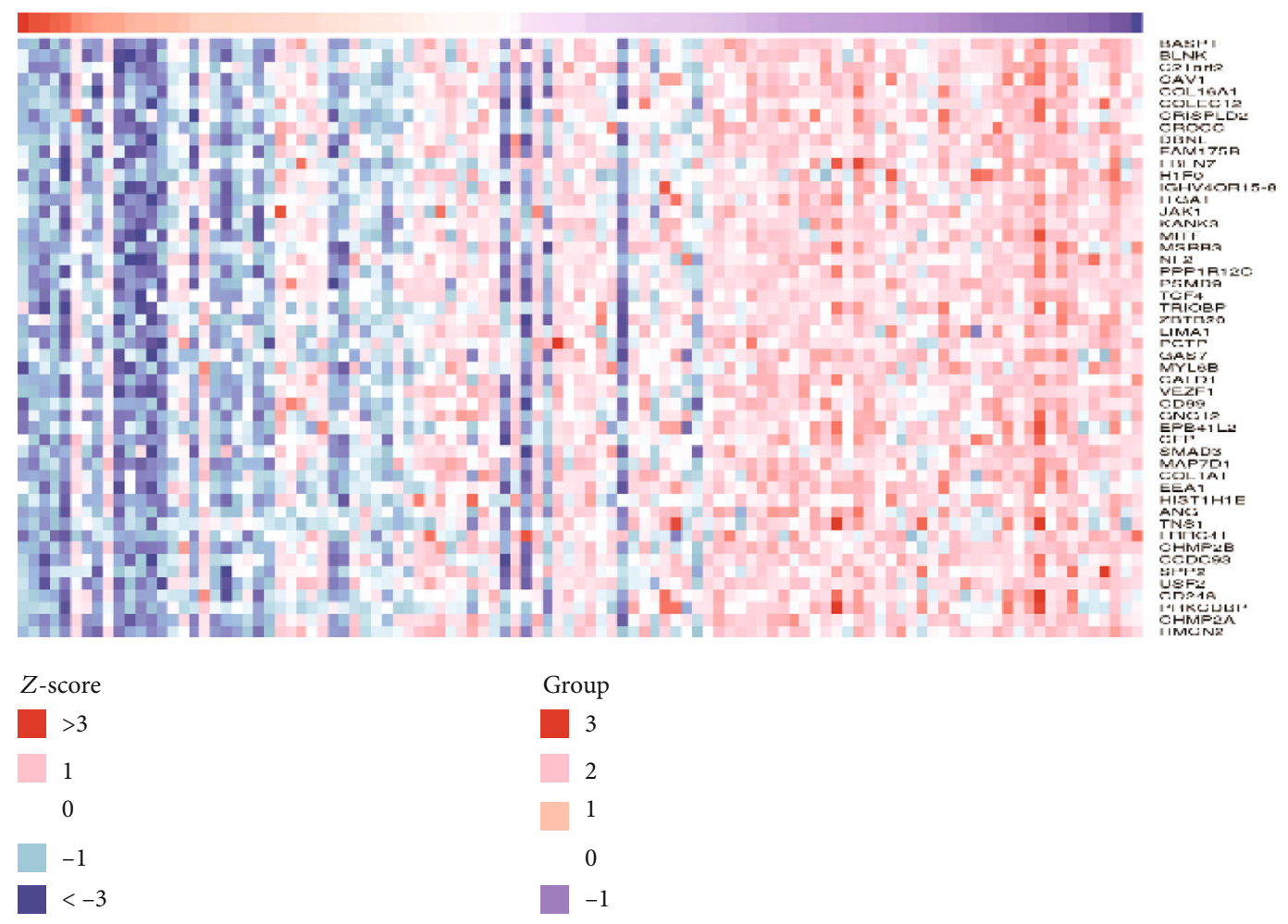

(c)

FIGURE 11: Genes differentially expressed in correlation with FLAD1 in breast invasive carcinoma (LinkedOmics). (a) Correlations between FLAD1 and genes differentially expressed in breast invasive carcinoma. (b, c) Heat maps showing genes positively and negatively correlated with FLAD1.

$0.01)$. The top 50 genes exhibiting positive or negative correlations with FLAD1 were evaluated in a heat map (Figures 11(b) and 11(c)).

The FLAD1 expression showed a strong positive association with the expression of $N L N$ (Spearman's correlation = $0.69, p<0.001$ ), UFC1 (Spearman's correlation $=0.68, p<$ 0.001 ), and UCHL5 (Spearman's correlation $=0.67, p<$ 0.001 ), which function in metalloendopeptidase activity and peptide binding, UFM1 transferase activity, and endopeptidase inhibitor activity.

We also conducted a KEGG analysis of positively and negatively correlated genes (Figures 12 (a) and 12(b), respectively) and found the enrichment for metabolic processes, biological regulation, nucleus, and protein binding.

\section{Discussion}

FLAD1 is related to the metabolism of water-soluble vitamins and cofactors, and FLAD1 mutations cause a FAD synthase deficiency, which is a rare genetic disease affecting mitochondrial energy metabolism and other riboflavin metabolism $[33,34]$. The overexpression of FLAD1 has been reported in various cancers such as hepatocellular carcinoma [8], gastric cancer [10], and breast cancer [11]. In this study, we analyzed the transcription levels of FLAD1 in pan-cancer analysis with a focus on breast cancer and further classified the results on the basis of the clinicopathologic parameters. These results provide evidences for FLAD1 as a new biomarker of breast cancer and suggest its clinical significance.

Based on extensive database mining, FLAD1 was found to be overexpressed in various cancers, including kidney renal papillary cell carcinoma, head-neck squamous cell carcinoma, esophageal cancers, colon adenocarcinoma, cholangiocarcinoma, breast invasive carcinoma, bladder urothelial carcinoma, and liver hepatocellular carcinoma. Amplification was the most frequent FLAD1 alteration type identified in breast cancer. We observed higher FLAD1 expression in the $\mathrm{ER}^{-}$and $\mathrm{PR}^{-}$group, HER2 ${ }^{+} \mathrm{NPI}$ stage 3, basal-like group, and triple-negative group. ER has two forms, $\alpha$ and $\beta$, which are encoded by ESR1 and ESR2, respectively [35]. PR also has two different isoforms, PRA and PRB, encoded by the same $P R$ gene [36]. Since ER-positive patients are eligible for hormonal therapy [37], ER status plays an important role in treatment decisions. A study based on the SEER database illustrated that patients with $\mathrm{ER}^{+} \mathrm{PR}^{+}$status had better survival than those who with an $\mathrm{ER}^{-} \mathrm{PR}^{-}$in each stage and age group [38]. In addition, patients with $\mathrm{ER}^{+} \mathrm{PR}^{+}$breast cancer respond better to tamoxifen than $\mathrm{ER}^{+} \mathrm{PR}^{-}$patients [39]. In our study, the FLAD1 expression was correlated with $\mathrm{ER}^{-}$ and $\mathrm{PR}^{-}$, which are related to poorer survival. The basallike group showed higher FLAD1 expression than that in other groups. Previous studies have shown that patients with basal-like cancers have significantly worse OS and recurrence-free survival than those of their luminal A counterparts [40-42]. Similar results have been found in a breast 

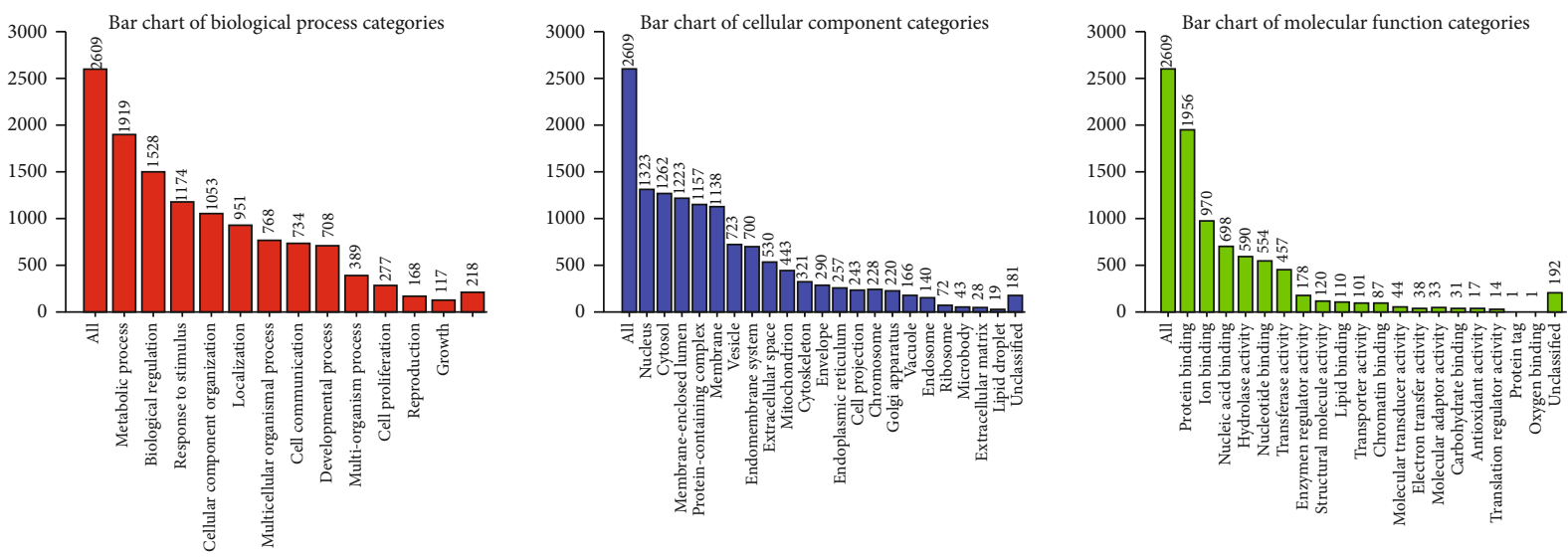

(a)
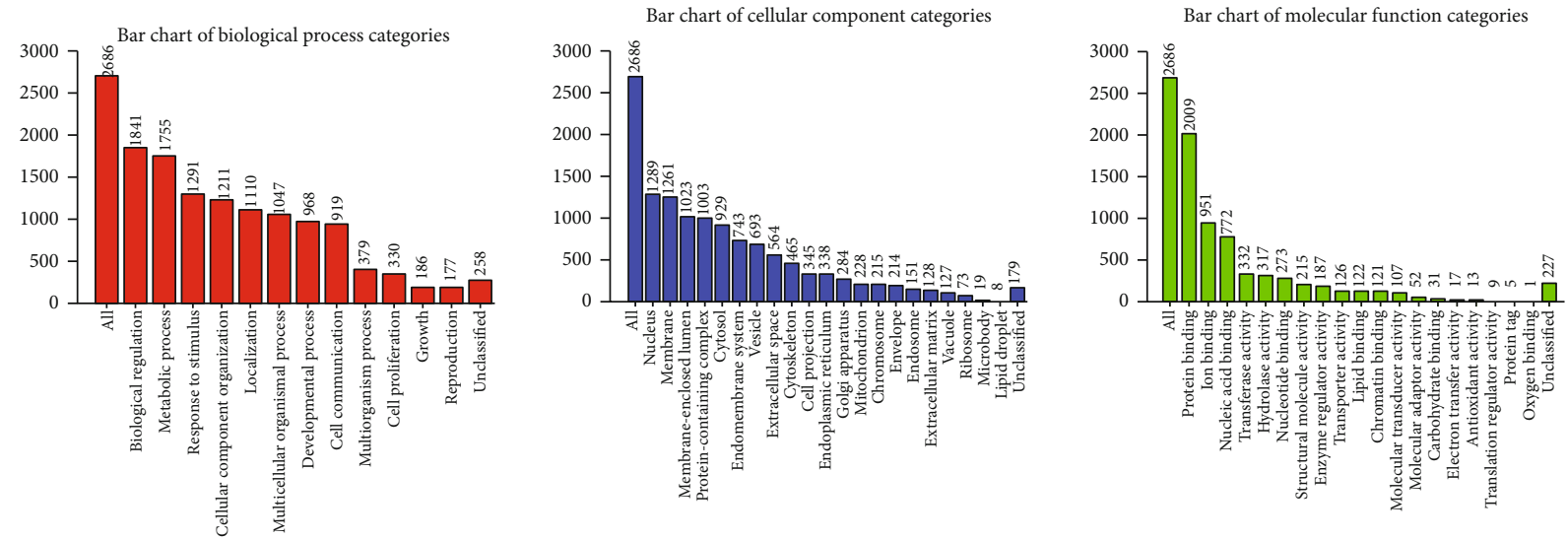

(b)

FIGURE 12: Bar charts showed KEGG pathway analysis of FLAD1 corrected genes (Linkomics). (a) KEGG pathway analysis of positive correlated genes. (B) KEGG pathway analysis of negative correlated genes.

cancer-specific survival analysis [43]. Triple-negative breast cancer lacks a therapeutic target owing to its negative profile for PR, ER, and HER2 [44]. Triple-negative breast cancer has a lower five-year survival rate compared with those of other breast cancers [45]. FLAD1 was also related to nodal status, which is a practical parameter for estimating prognosis [46].

We further analyzed clinical parameters in breast invasive carcinoma. The expression levels were higher in patients who were 21-40 years old than at those from other age groups and were higher in the African-American group than in the Caucasian group. In addition, the FLAD1 expression levels in the luminal subclass were significantly lower than those in the HER2-positive and triple-negative groups.

We also analyzed the significance and potential clinical application of FLAD1-related miRNAs. In previous studies, the FLAD1 expression was found to be linked to let-7b with respect to tumorigenesis in breast cancer, based on the analysis of somatic single-nucleotide variants and miRNAmRNA pairs [47]. In the present study, we found some FLAD1-related miRNAs, which showed significant differences in the cancer and normal samples, indicating their potential prognostic value.

We also found that the expression of FLAD1 in breast cancer is associated with the expression of genes involved in metabolic processes, including NLN, UFC1, and UCHL5. Among these, UCHL5 is reversibly recruited and activated by the $19 \mathrm{~S}$ proteasome and shows potential as a novel target for anticancer therapy [48]. Further research on the possibility of the application of FLAD1 as a therapeutic target based on small-molecule probes has yielded initial results [49], indicating that FLAD1 also has certain potential as a target for cancer treatment.

This study reports the significance of FLAD1 in cancer based on multilevel data in public databases and provides evidence for its potential as a biomarker for the diagnosis and prognosis of various cancers. One of the limitations of this study is its retrospective nature, because the analysis was only based on current databases and did not involve any prospective research for validation. In addition, this study mainly relies on bioinformatics, without a summary of detailed clinical information. Therefore, further research is needed to validate the results of this study and elucidate the biological mechanism underlying the role of FLAD1 in cancers.

\section{Data Availability}

The datasets in this study can be obtained from the listed database (Oncomine, cBioPortal, Breast cancer Gene- 
Expression Miner, UALCAN, GEO, BCIP, TNMplot, ENCORI, Kaplan-Meier Plotter and LinkedOmics).

\section{Conflicts of Interest}

The authors declare that they have no conflicts of interest.

\section{Acknowledgments}

We are grateful to all the researchers in Oncomine, cBioPortal, Breast Cancer Gene-Expression Miner, UALCAN, GEO, BCIP, TNMplot, ENCORI, Kaplan-Meier Plotter, and LinkedOmics. This study was funded by the National Natural Science Foundation of China (81970184, U1904139) and Department of Science and Technology of Henan Province (182102310114).

\section{Supplementary Materials}

Figure S1: survival analysis of positively related miRNAs. (a) hsa-miR-299-5p, (b) hsa-miR-154, (c) hsa-miR-299-3p, (d) hsa-miR-31, (e) hsa-miR-328, (f) hsa-miR-654-5p, and (g) hsa-miR-543. Figure S2: survival analysis of negatively correlated miRNAs. (Supplementary Materials)

\section{References}

[1] N. Harbeck and M. Gnant, "Breast cancer," The Lancet, vol. 389, no. 10074, pp. 1134-1150, 2017.

[2] F. Bray, J. Ferlay, I. Soerjomataram, R. L. Siegel, L. A. Torre, and A. Jemal, "Global cancer statistics 2018: GLOBOCAN estimates of incidence and mortality worldwide for 36 cancers in 185 countries," CA: a cancer journal for clinicians, vol. 68, no. 6, pp. 394-424, 2018.

[3] S. W. Lam, C. R. Jimenez, and E. Boven, "Breast cancer classification by proteomic technologies: current state of knowledge," Cancer treatment reviews, vol. 40, no. 1, pp. 129-138, 2014.

[4] Y. He, X. Li, Y. Meng et al., "A prognostic 11 long noncoding RNA expression signature for breast invasive carcinoma," Journal of Cellular Biochemistry, vol. 120, no. 10, pp. 1669216702, 2019.

[5] C. Brizio, M. Galluccio, R. Wait et al., "Over-expression in Escherichia coli and characterization of two recombinant isoforms of human FAD synthetase," Biochemical and biophysical research communications, vol. 344, no. 3, pp. 1008-1016, 2006.

[6] G. Stelzer, N. Rosen, I. Plaschkes et al., "The GeneCards suite: from gene data mining to disease genome sequence analyses," Current Protocols in Bioinformatics, vol. 54, no. 1, 2016.

[7] R. K. J. Olsen, E. Koňaříková, T. A. Giancaspero et al., "Riboflavin-responsive and -non-responsive mutations in FAD synthase cause multiple acyl-CoA dehydrogenase and combined respiratory-chain deficiency," American journal of human genetics, vol. 98, no. 6, pp. 1130-1145, 2016.

[8] C. Ye, X. Zhang, X. Chen et al., "Multiple novel hepatocellular carcinoma signature genes are commonly controlled by the master pluripotency factor OCT4," Cellular Oncology (Dordrecht), vol. 43, no. 2, pp. 279-295, 2020.

[9] R. Mitra, J. Lee, J. Jo et al., "Prediction of postoperative recurrence-free survival in non-small cell lung cancer by using an internationally validated gene expression model," Clinical cancer research, vol. 17, no. 9, pp. 2934-2946, 2011.

[10] P. Hu, Y. Pan, C. Wang et al., "FLAD1 is up-regulated in gastric cancer and is a potential prediction of prognosis," International Journal of Medical Sciences, vol. 17, no. 12, pp. 17631772, 2020.

[11] X. Jia, C. Wang, H. Huang, P. Zhang, Z. Yao, and L. Xu, "FLAD1 is overexpressed in breast cancer and is a potential predictor of prognosis and treatment," International Journal of Clinical and Experimental Medicine, vol. 12, pp. 31383152, 2019.

[12] D. R. Rhodes, J. Yu, K. Shanker et al., "ONCOMINE: A Cancer Microarray Database and Integrated Data-Mining Platform," Neoplasia, vol. 6, no. 1, pp. 1-6, 2004.

[13] J. Gao, B. A. Aksoy, U. Dogrusoz et al., "Integrative analysis of complex cancer genomics and clinical profiles using the cBioPortal," Science signaling, vol. 6, no. 269, p. pl1, 2013.

[14] E. Cerami, J. Gao, U. Dogrusoz et al., "The cBio cancer genomics portal: an open platform for exploring multidimensional cancer genomics data," Cancer discovery, vol. 2, no. 5, pp. 401404, 2012.

[15] P. Jezequel, J. S. Frenel, L. Campion et al., "bc-GenExMiner 3.0: new mining module computes breast cancer gene expression correlation analyses," Database, vol. 2013, 2013.

[16] P. Jézéquel, M. Campone, W. Gouraud et al., "bc-GenExMiner: an easy-to-use online platform for gene prognostic analyses in breast cancer," Breast cancer research and treatment, vol. 131, no. 3, pp. 765-775, 2012.

[17] P. Jézéquel, W. Gouraud, F. Ben Azzouz et al., "bc-GenExMiner 4.5: new mining module computes breast cancer differential gene expression analyses," Database, vol. 2021, 2021.

[18] J.-H. Li, S. Liu, H. Zhou, L.-H. Qu, and J.-H. Yang, "starBase v2.0: decoding miRNA-ceRNA, miRNA-ncRNA and protein-RNA interaction networks from large-scale CLIP-Seq data," Nucleic acids research, vol. 42, no. D1, pp. D92-D97, 2014.

[19] P. Shannon, A. Markiel, O. Ozier et al., "Cytoscape: a software environment for integrated models of biomolecular interaction networks," Genome Research, vol. 13, no. 11, pp. 2498 2504, 2003.

[20] Á. Bartha and B. Győrffy, “TNMplot.com: a web tool for the comparison of gene expression in normal, tumor and metastatic tissues," International Journal of Molecular Sciences, vol. 22, no. 5, 2021.

[21] D. S. Chandrashekar, B. Bashel, S. A. H. Balasubramanya et al., "UALCAN: a portal for facilitating tumor subgroup gene expression and survival analyses," Neoplasia, vol. 19, no. 8, pp. 649-658, 2017.

[22] J. Wu, S. Hu, Y. Chen et al., "BCIP: a gene-centered platform for identifying potential regulatory genes in breast cancer," Scientific Reports, vol. 7, no. 1, 2017.

[23] B. Györffy, A. Lanczky, A. C. Eklund et al., "An online survival analysis tool to rapidly assess the effect of 22,277 genes on breast cancer prognosis using microarray data of 1,809 patients," Breast cancer research and treatment, vol. 123, no. 3, pp. 725-731, 2010.

[24] S. V. Vasaikar, P. Straub, J. Wang, and B. Zhang, "LinkedOmics: analyzing multi-omics data within and across 32 cancer types," Nucleic acids research, vol. 46, no. D1, pp. D956-d963, 2018.

[25] T. Casey, J. Bond, S. Tighe et al., "Molecular signatures suggest a major role for stromal cells in development of invasive breast 
cancer," Breast cancer research and treatment, vol. 114, no. 1, pp. 47-62, 2009.

[26] R. Z. Liu, K. Graham, D. D. Glubrecht, D. R. Germain, J. R. Mackey, and R. Godbout, "Association of FABP5 expression with poor survival in triple-negative breast cancer: implication for retinoic acid therapy," The American Journal of Pathology, vol. 178, no. 3, pp. 997-1008, 2011.

[27] T. Z. Tan, Q. H. Miow, Y. Miki et al., "Epithelial-mesenchymal transition spectrum quantification and its efficacy in deciphering survival and drug responses of cancer patients," $E M B O$ Molecular Medicine, vol. 6, no. 10, pp. 1279-1293, 2014.

[28] METABRIC Group, C. Curtis, S. P. Shah et al., "The genomic and transcriptomic architecture of 2,000 breast tumours reveals novel subgroups," Nature, vol. 486, no. 7403, pp. 346-352, 2012.

[29] K. Yu, K. Ganesan, L. K. Tan et al., “A precisely regulated gene expression cassette potently modulates metastasis and survival in multiple solid cancers," PLoS Genetics, vol. 4, no. 7, article e1000129, 2008.

[30] Y. Pawitan, J. Bjöhle, L. Amler et al., “Gene expression profiling spares early breast cancer patients from adjuvant therapy: derived and validated in two population-based cohorts," Breast cancer research : BCR, vol. 7, no. 6, pp. R953-R964, 2005.

[31] C. Desmedt, F. Piette, S. Loi et al., "Strong time dependence of the 76-gene prognostic signature for node-negative breast cancer patients in the TRANSBIG multicenter independent validation series," Clinical cancer research, vol. 13, no. 11, pp. 3207-3214, 2007.

[32] L. D. Miller, J. Smeds, J. George et al., “An expression signature for p53 status in human breast cancer predicts mutation status, transcriptional effects, and patient survival," Proceedings of the National Academy of Sciences of the United States of America, vol. 102, no. 38, pp. 13550-13555, 2005.

[33] Y. Yildiz, R. K. J. Olsen, H. S. Sivri, Z. Akcoren, H. H. Nygaard, and A. Tokatli, "Post-mortem detection of FLAD1 mutations in 2 Turkish siblings with hypotonia in early infancy," Neuromuscular disorders : NMD, vol. 28, no. 9, pp. 787-790, 2018.

[34] A. Kuroda and J. Sekiguchi, "High-level transcription of the major Bacillus subtilis autolysin operon depends on expression of the sigma D gene and is affected by a sin (flaD) mutation," Journal of bacteriology, vol. 175, no. 3, pp. 795-801, 1993.

[35] L. Bjornstrom and M. Sjoberg, "Mechanisms of estrogen receptor signaling: convergence of genomic and nongenomic actions on target genes," Molecular endocrinology, vol. 19, no. 4, pp. 833-842, 2005.

[36] C. H. Yip and A. Rhodes, "Estrogen and progesterone receptors in breast cancer," Future oncology, vol. 10, no. 14, pp. 2293-2301, 2014.

[37] I. A. Olivotto, P. T. Truong, C. H. Speers et al., "Time to stop progesterone receptor testing in breast cancer management," Journal of clinical oncology, vol. 22, no. 9, pp. 1769-1770, 2004.

[38] V. R. Grann, A. B. Troxel, N. J. Zojwalla, J. S. Jacobson, D. Hershman, and A. I. Neugut, "Hormone receptor status and survival in a population-based cohort of patients with breast carcinoma," Cancer, vol. 103, no. 11, pp. 2241-2251, 2005.

[39] X. Cui, R. Schiff, G. Arpino, C. K. Osborne, and A. V. Lee, "Biology of progesterone receptor loss in breast cancer and its implications for endocrine therapy," Journal of clinical oncology, vol. 23, no. 30, pp. 7721-7735, 2005.
[40] T. Sorlie, C. M. Perou, R. Tibshirani et al., "Gene expression patterns of breast carcinomas distinguish tumor subclasses with clinical implications," Proceedings of the National Academy of Sciences of the United States of America, vol. 98, no. 19, pp. 10869-10874, 2001.

[41] T. Sørlie, R. Tibshirani, J. Parker et al., "Repeated observation of breast tumor subtypes in independent gene expression data sets," Proceedings of the National Academy of Sciences of the United States of America, vol. 100, no. 14, pp. 8418-8423, 2003.

[42] S. Calza, P. Hall, G. Auer et al., "Intrinsic molecular signature of breast cancer in a population-based cohort of 412 patients," Breast cancer research : BCR, vol. 8, no. 4, p. R34, 2006.

[43] L. A. Carey, C. M. Perou, C. A. Livasy et al., "Race, breast cancer subtypes, and survival in the Carolina breast Cancer study," Journal of the American Medical Association, vol. 295, no. 21, pp. 2492-2502, 2006.

[44] A. A. Jitariu, A. M. Cimpean, D. Ribatti, and M. Raica, "Triple negative breast cancer: the kiss of death," Oncotarget, vol. 8, no. 28, pp. 46652-46662, 2017.

[45] R. Dent, M. Trudeau, K. I. Pritchard et al., "Triple-negative breast cancer: clinical features and patterns of recurrence," Clinical cancer research, vol. 13, no. 15, pp. 4429-4434, 2007.

[46] C. L. Carter, C. Allen, and D. E. Henson, "Relation of tumor size, lymph node status, and survival in 24,740 breast cancer cases," Cancer, vol. 63, no. 1, pp. 181-187, 1989.

[47] C. Li, B. Wu, H. Han, J. Zhao, Y. Bai, and X. Liu, "Identification of microRNA-related tumorigenesis variants and genes in The Cancer Genome Atlas (TCGA) Data," Genes, vol. 11, no. 9, p. 953, 2020.

[48] X. Xia, Y. Liao, Z. Guo et al., “Targeting proteasome-associated deubiquitinases as a novel strategy for the treatment of estrogen receptor-positive breast cancer," Oncogene, vol. 7, no. 9, p. 75, 2018.

[49] K. Cheng, J. S. Lee, P. Hao, S. Q. Yao, K. Ding, and Z. Li, “Tetrazole-based probes for integrated phenotypic screening, affinity-based proteome profiling, and sensitive detection of a cancer biomarker," Angewandte Chemie (International Ed. in English), vol. 56, no. 47, pp. 15044-15048, 2017. 